\title{
Identification of Aerodynamic Damping Matrix for Operating Wind Turbines
}

Chao Chen ${ }^{1,2^{*}}$, Philippe Duffour ${ }^{2}, \mathrm{Kaoshan}_{\mathrm{Dai}^{3}}{ }^{3}$ Ying Wang ${ }^{4}$, and Paul Fromme ${ }^{5}$

${ }^{1}$ Key Laboratory for Wind and Bridge Engineering, Hunan University, China

${ }^{2}$ Department of Civil, Environmental and Geomatic Engineering, University College

London, United Kingdom

${ }^{3}$ Department of Civil Engineering, Sichuan University, China

${ }^{4}$ College of Civil Engineering, Tongji University, China

${ }^{5}$ Department of Mechanical Engineering, University College London, United Kingdom

*Corresponding author: steinchen@hnu.edu.cn 


\title{
Identification of Aerodynamic Damping Matrix for Operating Wind Turbines
}

\begin{abstract}
Accurate knowledge of wind turbine tower vibration damping is essential for the estimation of fatigue life. However, the responses in the fore-aft and side-side directions are coupled through the wind-rotor interaction under operational conditions. This causes energy transfers and complicates aerodynamic damping identification using conventional damping ratios. Employing a reduced two-degree freedom wind turbine model developed in this paper, this coupling can be accurately expressed by an unconventional aerodynamic damping matrix. Simulated time series obtained from this model were successfully verified against the outputs from the wind turbine simulation tool FAST. Based on the reduced system obtained, a matrix-based identification method is proposed to identify the aerodynamic damping for numerically simulated wind turbine tower responses. Applying harmonic excitations to the tower allowed the frequency response functions of the wind turbine system to be obtained and the aerodynamic damping matrix to be extracted. Results from this identification were compared to traditional operational modal analysis methods including standard and modified stochastic subspace identification. The damping ratios in the fore-aft direction were successfully identified by all methods, but results showed that the identified damping matrix performs better in capturing the aerodynamic damping and coupling for the side-side responses.
\end{abstract}

Key words: damping identification, wind turbine, frequency response function, aerodynamic damping, fatigue 


\section{Introduction}

As wind turbines become larger to harvest more wind energy, they become more flexible and susceptible to vibration. Due to vibration during their operational life, the tower, blades and other components are prone to fatigue damage, which needs to be carefully considered at the design stage or for the determination of remaining life ([1][2]). Damping is a key parameter in wind turbine systems as it limits the vibration amplitude. Therefore, identifying damping reliably in these systems is an important issue. Different damping sources, including structural, aerodynamic, soil, and hydrodynamic (for offshore wind turbines) damping, and damping devices (if installed), contribute to the total damping in wind turbines [3]. For wind turbines in operation, the fore-aft aerodynamic damping has the highest contribution, so the measured total damping could also be seen as a close substitute for the aerodynamic damping. In many published studies the distinction between total damping and aerodynamic damping for operating wind turbines is not always clear [4]. In practice only the total damping can be measured directly.

The identification of the aerodynamic damping is challenging because of the complex fluid-structure interaction and the nature of turbulent wind. Classical experimental modal analysis requires a controlled excitation to trigger a dynamic response [5]. Operational Modal Analysis (OMA), which does not require controlled force excitation of large wind turbine structures, is usually preferred to measure aerodynamic damping in operation. However, methods with artificial excitations (such as boat impact [6] and hydraulic shaker [7]) have also been used. In the literature, a limited number of studies focussed specifically on identifying this damping contribution ([4], [6-14]). Measuring damping in parked wind turbines has proved easier than in operating conditions [8]. Studies by researchers such as Devriendt et al. [9] and Bajrić et al. [10] have successfully identified damping for parked turbines using different identification methods either in the time or frequency domain. The identification methods used for operating turbines are similar to those for parked turbines. Hansen et al. [4] used Stochastic Subspace Identification (SSI) to extract the damping ratios for an operating wind turbine but the resulting damping ratios were scattered and the contribution due to aerodynamic damping alone remains unclear. Ozbek and Rixen [11] used the Least Square Complex Exponential (LSCE) method to identify aerodynamic damping with data from strain gauge and photogrammetry measurements. The measured damping ratio values did not 
vary significantly and were close to simulation results obtained using HAWCStab [12]. Devriendt et al. [13] used the poly Least-Squares Complex Frequency-domain (pLSCF) estimator to identify the total damping for an operational wind turbine. They emphasised that harmonics in the excitation would hinder the use of classical OMA methods which assumes that measured resonances are only caused by amplification of the broadband noise excitation at the natural frequencies of the system. Hu et al. [14] implemented the p-LSCF method to identify the total damping in an operating turbine with different rotation speeds. The resonance due to 3P loading was observed to have a significant effect on the identified total damping. Koukoura et al. [6] studied the total damping in an operating offshore wind turbine under ambient excitation using the Enhanced Frequency Domain Decomposition (EFDD) method. They found that a beating phenomenon observed on the autocorrelation function of the response made the identification less reliable, especially for side-side vibrations.

Although much research has been conducted to identify the aerodynamic damping in operating wind turbines using traditional OMA methods, limitations in these methods still exist. For operating wind turbines, the validity of some basic assumptions underpinning the implementation of most OMA methods remain doubtful and this causes difficulties in applying OMA techniques to wind turbines. These difficulties have been described by Tcherniak et al. [15] and Ozbek et al. [8]. First, the excitations to the structure need to be uncorrelated, but the forces exciting a wind turbine are not uncorrelated as they are coupled due to the influence of the rotor rotation. Second, traditional OMA methods assume that the resultant responses due to ambient excitation only include harmonics caused by the natural modes of the structure but not harmonics due to ambient excitations themselves. This assumption is violated by the rotor rotation causing $1 \mathrm{P}, 3 \mathrm{P}$ etc. loadings to the tower. Third, traditional OMA techniques require that the structure system itself is a time-invariant system, which is not the case for wind turbines as aerodynamic damping is influenced by the inflow wind speed, the rotation speed and the pitch angles. None of these parameters are constant for wind turbines in normal operation due to the stochastic nature of wind turbulence and the variability of controlled conditions [16]. Fourth, and maybe less important, the excitations caused by turbulent wind field are not white noise. The effort to extract dynamic parameters for wind turbines resulted in the development of modified OMA methods suitable for large wind turbine structures under ambient excitations. The Natural Excitation Technique (NExT) OMA method developed by James et al. [17] was initially used for modal 
parameter extraction of operating vertical-axis wind turbines. Some researchers improved the traditional OMA methods so that these methods are able to identify the wind turbine system when the excitations contain harmonics. Dai et al. [18] developed a modified SSI which is able to identify modal parameters of structures when the excitations contain harmonic components. They applied this method to a wind turbine tower and successfully obtained the damping ratios by distinguishing the natural modes and the harmonically excited modes. Dong et al. [19] applied a similar modified SSI method to identify the frequency and damping of an operating turbine, and obtained a wide range of values in different operating conditions.

Besides the difficulties mentioned above, all previously discussed studies assumed that the wind turbine system is decoupled in terms of the Fore-Aft (FA) and Side-Side (SS) motions. Recent research [20] showed that the rotating blades introduce significant damping coupling between the FA and SS directions for an operating wind turbine and this coupling is non-classical, i.e., the coupling is through a damping matrix which, unusually, is not symmetric. From this different way of looking at the aerodynamic damping, a new operational identification method can be developed that extracts the aerodynamic damping matrix directly. This article describes this approach applied to three-blade horizontal-axis wind turbines and is organised as follows. Section 2 describes the wind turbine model with the full aerodynamic damping matrix and the derivation that converts the original model with full aerodynamic damping matrix to a Two-Degree of Freedom (2-DOF) model. In Section 3, model verification against the wind turbine modelling package FAST [21] developed by NREL is presented and problems associated with traditional damping identification aimed at obtaining damping ratios are stated. Section 4 shows how the identification method based on the aerodynamic damping matrix performs for identification examples. Section 5 concludes the paper.

\section{Model description}

\subsection{Wind turbine model description}

A three-bladed onshore wind turbine was modelled using bespoke Finite Element (FE) code written in MATLAB. The model is a cantilever beam made of 11 Euler-Bernoulli beam elements that represent the tower and a lumped mass at the top representing the 
Rotor-Nacelle Assembly (RNA). Each node is associated with a translational and rotational degree of freedom in two perpendicular horizontal directions, $x$ (FA) and $y$ (SS) as shown in Figure 1. The model has 44 degrees of freedom in total. The geometric and material properties of the system are based on a modified NREL 5MW reference onshore wind turbine [22], with properties listed in Table 1. The modification consists in placing the centre of mass of the RNA at the tower top and setting the moments of inertia of the RNA relative to the tower top to zero. This was necessary as it is not clear how to specify different RNA moments of inertia in different directions in FAST.

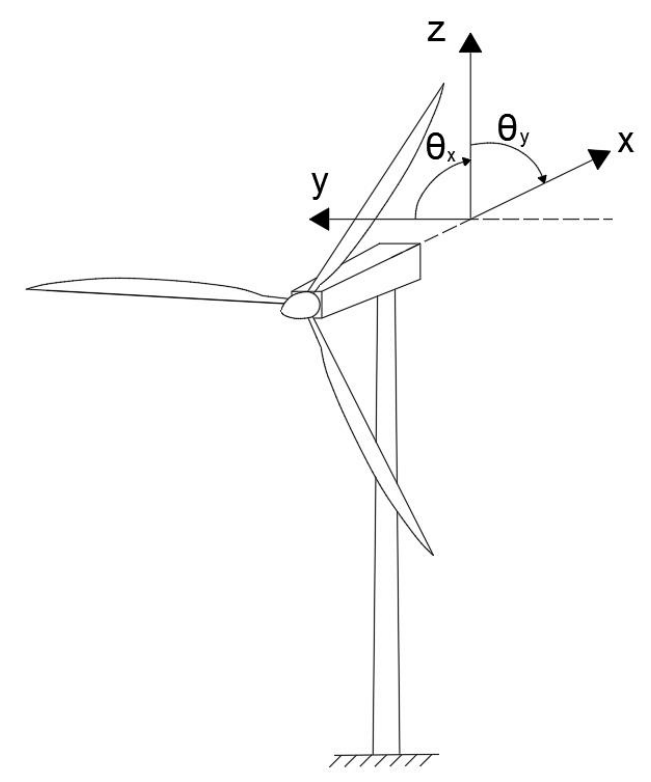

Figure 1. Schematic of the wind turbine.

Table 1. Properties of the modified NREL 5MW reference onshore wind turbine.

\begin{tabular}{|l|l|}
\hline Rotor Diameter, $R$ & $126 \mathrm{~m}$ \\
\hline Hub Height from MSL & $87.6 \mathrm{~m}$ \\
\hline Tower Diameter, $D$ & $3.87-6.00 \mathrm{~m}$ \\
\hline Tower Thickness, $t$ & $19-27 \mathrm{~mm}$ \\
\hline Lumped Mass at Top & $3.5 \times 10^{5} \mathrm{~kg}$ \\
\hline Rated Wind Speed & $12.1 \mathrm{~m} / \mathrm{s}$ \\
\hline Natural Frequency & $0.34 \mathrm{~Hz}$ \\
\hline
\end{tabular}

Using the information provided for the reference turbine, the mass and stiffness matrices of the turbine system were assembled. 
The equation of motion of the system is:

$$
\mathbf{M} \ddot{\mathbf{u}}(t)+\mathbf{C}_{\text {Struc }} \dot{\mathbf{u}}(t)+\mathbf{K u}(t)=\mathbf{F}^{\text {Flex }}(t),
$$

where $\mathbf{M}, \mathbf{C}_{\text {Struc }}$ and $\mathbf{K}$ are the mass, structural damping and stiffness matrices respectively. $\mathbf{C}_{\text {Struc }}$ is assumed to be a proportional damping matrix.

$$
\mathbf{F}_{\text {Flex }}^{\text {Top }}(t)=\left[\mathrm{F}_{x}^{\text {Flex }}(t) \mathrm{F}_{y}^{\text {Flex }}(t) \mathrm{M}_{x}^{\text {Flex }}(t) \mathrm{M}_{y}^{\text {Flex }}(t)\right]^{\mathrm{T}} \text {. }
$$

Given the relative wind speeds acting on the blade elements, the resultant aerodynamic force $\mathbf{F}_{\text {Flex }}^{\text {Top }}(t)$ can be calculated using Blade Element Momentum (BEM) Theory [23]. As described in [20], $\mathbf{F}_{\text {Flex }}^{\text {Top }}(t)$ can be linearized with respect to the tower top velocities. This gives a first term $\mathbf{F}_{\text {Rigid }}^{\text {Top }}(t)$ independent of the velocities which represents the aerodynamic force applied to a rigid tower, plus a second term linear in velocities which can be expressed using an aerodynamic damping matrix $\mathbf{C}_{\text {Aero }}$ :

$$
\mathbf{F}_{\text {Flex }}^{\text {Top }}(t)=\mathbf{F}_{\text {Rigid }}^{\text {Top }}(t)-\mathbf{C}_{\text {Aero }} \dot{\mathbf{u}}^{\text {Top }}(t) .
$$

The derivation of aerodynamic damping matrix in [20] requires the initial assumption that the inflow wind field is constant. As shown in Appendix A, an extension to this derivation can be made to consider a turbulent and non-uniform wind field, which leads to an aerodynamic damping matrix $\mathbf{C}_{\text {Aero }}$ in the following form:

$$
\mathbf{C}_{\text {Aero }}=\left[\begin{array}{cccc}
c_{x x} & c_{x y} & c_{x \theta_{x}} & c_{x \theta_{y}} \\
c_{y x} & c_{y y} & c_{y \theta_{x}} & c_{y \theta_{y}} \\
c_{\theta_{x} x} & c_{\theta_{x} y} & c_{\theta_{x} \theta_{x}} & c_{\theta_{x} \theta_{y}} \\
c_{\theta_{y} x} & c_{\theta_{y} y} & c_{\theta_{y} \theta_{x}} & c_{\theta_{y} \theta_{y}}
\end{array}\right],
$$

where the matrix components $c_{i j}$ are defined analytically in Appendix A in terms of derivatives of the blade element forces.

The linearized equation of motion for the FE model considering aerodynamic coupling is

$$
\mathbf{M} \ddot{\mathbf{u}}(t)+\mathbf{C u}(t)+\mathbf{K u}(t)=\mathbf{F}^{\text {Rigid }}(t),
$$

where $\mathbf{C}$ is $\mathbf{C}_{\text {Struc }}$ plus the terms of $\mathbf{C}_{\text {Aero }}$ added at the relevant locations and $\mathbf{F}^{\text {Rigid }}(t)$ is the tower top force $\mathbf{F}_{\text {Rigid }}^{\text {Top }}(t)$ padded with zeros for all other degree of freedoms.

\subsection{Reduction to a 2-DOF system}

The response of the system expressed in Equation (1) can be calculated using the concept of modal decomposition, when $\mathbf{C}_{S t r u c}$ is assumed to be a proportional damping 
matrix and $\mathbf{F}^{\text {Flex }}(t)$ is initially regarded as an external force independent of the dynamic properties of the system. In wind turbines, the FA and SS responses are dominated by the FA and SS first bending modes so only two related generalised coordinates will be considered [24] in the following derivation. Expressing the response as modal superpositions and pre-multiplying Eq. (3) by the transpose of each mode shape in turn, the equations of motion for the first FA and SS bending modes can be written as:

$$
\begin{aligned}
& \bar{m}_{x} \ddot{\alpha}_{x}(t)+2 \bar{\zeta}_{x} \sqrt{\bar{m}_{x} \bar{k}_{x}} \dot{\alpha}_{x}(t)+\bar{k}_{x} \alpha_{x}(t)=\boldsymbol{\phi}_{x}^{\mathrm{T}} \mathbf{F}_{x}^{\text {Flex }}(t) \\
& \bar{m}_{y} \ddot{\alpha}_{y}(t)+2 \bar{\zeta}_{y} \sqrt{\bar{m}_{y} \bar{k}_{y}} \dot{\alpha}_{y}(t)+\bar{k}_{y} \alpha_{y}(t)=\boldsymbol{\phi}_{y}^{\mathrm{T}} \mathbf{F}_{y}^{\text {Flex }}(t)
\end{aligned}
$$

where $\bar{m}_{x}, \bar{m}_{y}, \bar{k}_{x}$ and $\bar{k}_{y}$ are the modal mass and stiffness for the first FA/SS mode respectively, $\bar{\zeta}_{x}$ and $\bar{\zeta}_{y}$ are the structural modal damping ratios, and $\alpha_{x}(t)$ and $\alpha_{y}(t)$ are the generalised coordinates for the FA and SS directions respectively. It should be noted that the employed modal properties are for the "undamped modes" as these properties are calculated from the mass and stiffness matrices in Equation (3), excluding the damping matrix. The actual modes of the entire system including the nonsymmetric damping matrix are complex [25], but we employ the undamped modes as a mathematical tool to format the problem for later identification. Structural damping terms were considered in Equation (4) and the following derivations to demonstrate how the developed 2-DOF model includes these terms for a complete wind turbine model. For the results showing the identification of aerodynamic damping, zero structural damping is later assumed throughout. The modal forces applied in the FA and SS directions after modal decomposition are $\mathbf{F}_{x}^{\text {Flex }}(t)=\left[\mathrm{F}_{x}^{\text {Flex }}(t) \mathrm{M}_{y}^{\text {Flex }}(t)\right]^{\mathrm{T}}$ and $\mathbf{F}_{y}^{\text {Flex }}(t)=\left[\mathrm{F}_{y}^{\text {Flex }}(t) \mathrm{M}_{x}^{\text {Flex }}(t)\right]^{\mathrm{T}}$ multiplied by the truncated mode shapes. The truncated mode shapes for the first FA and SS bending modes of the system in Equation (4) are $\boldsymbol{\phi}_{x}=\left[\phi_{x 1} \phi_{x 2}\right]^{\mathrm{T}}$ and $\boldsymbol{\phi}_{y}=\left[\phi_{y 1} \phi_{y 2}\right]^{\mathrm{T}}$, where $\phi_{x 1}$ and $\phi_{y 1}$ correspond to the displacement motion while $\phi_{x 2}$ and $\phi_{y 2}$ correspond to the angular motion for the first FA and SS bending modes respectively. The displacement and angular motions at the tower top can be expressed by multiplying the mode shapes and generalised coordinates, i.e., $\dot{u}_{x} \approx \phi_{x 1} \dot{\alpha}_{x}(t), \dot{u}_{y} \approx \phi_{y 1} \dot{\alpha}_{y}(t), \dot{\theta}_{x} \approx \phi_{y 2} \dot{\alpha}_{x}(t)$ and $\dot{\theta}_{y} \approx \phi_{x 2} \dot{\alpha}_{x}(t)$ for the velocities. Since $\mathbf{F}_{\text {Flex }}^{\text {Top }}(t)=\mathbf{F}_{\text {Rigid }}^{\text {Top }}(t)-\mathbf{C}_{\text {Aero }} \dot{\mathbf{u}}^{\text {Top }}(t)$, the modal force in the FA direction, $\boldsymbol{\phi}_{x}^{\mathrm{T}} \mathbf{F}_{x}^{\text {Flex }}(t)$, can be written as 


$$
\begin{aligned}
\boldsymbol{\phi}_{x}^{\mathrm{T}} \mathbf{F}_{x}^{F l e x}(t)= & {\left[\begin{array}{ll}
\phi_{x 1} & \phi_{x 2}
\end{array}\right]\left\{\left[\begin{array}{c}
F_{x}^{\text {Rigid }}(t) \\
\mathrm{M}_{y}^{\text {Rigid }}(t)
\end{array}\right]\right.} \\
& \left.-\left[\begin{array}{cccc}
c_{x x} & c_{x y} & c_{x \theta_{x}} & c_{x \theta_{y}} \\
c_{\theta_{y} x} & c_{\theta_{y} y} & c_{\theta_{y} \theta_{x}} & c_{\theta_{y} \theta_{y}}
\end{array}\right]\left[\begin{array}{l}
\phi_{x 1} \dot{\alpha}_{x}(t) \\
\phi_{y 1} \dot{\alpha}_{y}(t) \\
\phi_{y 2} \dot{\alpha}_{y}(t) \\
\phi_{x 2} \dot{\alpha}_{x}(t)
\end{array}\right]\right\} .
\end{aligned}
$$

The above equation can be simplified to

$$
\begin{aligned}
& \boldsymbol{\phi}_{x}^{\mathrm{T}} \mathbf{F}_{x}^{F l e x}(t)=\phi_{x 1} F_{x}^{\text {Rigid }}(t)+\phi_{x 2} M_{y}^{\text {Rigid }}(t) \\
& -\left(\phi_{x 1}^{2} c_{x x}+\phi_{x 1} \phi_{x 2} c_{x \theta_{y}}+\phi_{x 2} \phi_{x 1} c_{\theta_{y} x}+\phi_{x 2}^{2} c_{\theta_{y} \theta_{y}}\right) \dot{\alpha}_{x}(t) \\
& -\left(\phi_{x 1} \phi_{y 1} c_{x y}+\phi_{x 1} \phi_{y 2} c_{x \theta_{x}}+\phi_{x 2} \phi_{y 1} c_{\theta_{y} y}+\phi_{x 2} \phi_{y 2} c_{\theta_{y} \theta_{x}}\right) \dot{\alpha}_{y}(t) .
\end{aligned}
$$

Similarly, the corresponding SS modal force is

$$
\begin{aligned}
& \boldsymbol{\phi}_{y}^{\mathrm{T}} \mathbf{F}_{y}^{\text {Flex }}(t)=\phi_{y 1} F_{y}^{\text {Rigid }}(t)+\phi_{y 2} M_{x}^{\text {Rigid }}(t) \\
& -\left(\phi_{y 1} \phi_{x 1} c_{y x}+\phi_{y 1} \phi_{x 2} c_{y \theta_{y}}+\phi_{y 2} \phi_{x 1} c_{\theta_{x} x}+\phi_{y 2} \phi_{x 2} c_{\theta_{x} \theta_{y}}\right) \dot{\alpha}_{x}(t) \\
& -\left(\phi_{y 1}^{2} c_{y y}+\phi_{y 1} \phi_{y 2} c_{y \theta_{x}}+\phi_{y 2} \phi_{y 1} c_{\theta_{x}}+\phi_{y 2}^{2} c_{\theta_{x}} \theta_{x}\right) \dot{\alpha}_{y}(t) .
\end{aligned}
$$

Therefore, the equations of motion for the first bending modes in FA and SS directions can be written in matrix form:

$$
\begin{gathered}
{\left[\begin{array}{cc}
\bar{m}_{x} & 0 \\
0 & \bar{m}_{y}
\end{array}\right]\left[\begin{array}{l}
\ddot{\alpha}_{x}(t) \\
\ddot{\alpha}_{y}(t)
\end{array}\right]+\left[\begin{array}{cc}
\bar{c}_{x x}+\bar{s}_{x} & \bar{c}_{x y} \\
\bar{c}_{y x} & \bar{c}_{y y}+\bar{s}_{y}
\end{array}\right]\left[\begin{array}{l}
\dot{\alpha}_{x}(t) \\
\dot{\alpha}_{y}(t)
\end{array}\right]} \\
+\left[\begin{array}{cc}
\bar{k}_{x} & 0 \\
0 & \bar{k}_{y}
\end{array}\right]\left[\begin{array}{l}
\alpha_{x}(t) \\
\alpha_{y}(t)
\end{array}\right]=\left[\begin{array}{l}
\phi_{x 1} F_{x}^{\text {Rigid }}(t)+\phi_{x 2} M_{y}^{\text {Rigid }}(t) \\
\phi_{y 1} F_{y}^{\text {Rigid }}(t)+\phi_{y 2} M_{x}^{\text {Rigid }}(t)
\end{array}\right],
\end{gathered}
$$

where

$$
\begin{gathered}
\bar{c}_{x x}=\phi_{x 1}^{2} c_{x x}+\phi_{x 1} \phi_{x 2} c_{x \theta_{y}}+\phi_{x 2} \phi_{x 1} c_{\theta_{y} x}+\phi_{x 2}^{2} c_{\theta_{y} \theta_{y}}, \\
\bar{c}_{x y}=\phi_{x 1} \phi_{y 1} c_{x y}+\phi_{x 1} \phi_{y 2} c_{x \theta_{x}}+\phi_{x 2} \phi_{y 1} c_{\theta_{y} y}+\phi_{x 2} \phi_{y 2} c_{\theta_{y} \theta_{x}}, \\
\bar{c}_{y x}=\phi_{y 1} \phi_{x 1} c_{y x}+\phi_{y 1} \phi_{x 2} c_{y \theta_{y}}+\phi_{y 2} \phi_{x 1} c_{\theta_{x} x}+\phi_{y 2} \phi_{x 2} c_{\theta_{x} \theta_{y}}, \\
\bar{c}_{y y}=\phi_{y 1}^{2} c_{y y}+\phi_{y 1} \phi_{y 2} c_{y \theta_{x}}+\phi_{y 2} \phi_{y 1} c_{\theta_{x} y}+\phi_{y 2}^{2} c_{\theta_{x} \theta_{x}}, \\
\bar{s}_{x}=2 \bar{\zeta}_{x} \sqrt{\bar{m}_{x} \bar{k}_{x}} \\
\bar{s}_{y}=2 \bar{\zeta}_{y} \sqrt{\bar{m}_{y} \bar{k}_{y} .}
\end{gathered}
$$


Here the modal damping matrix $\overline{\mathbf{C}}$ is defined as

$$
\overline{\mathbf{C}}=\left[\begin{array}{cc}
\bar{c}_{x x}+\bar{s}_{x} & \bar{c}_{x y} \\
\bar{c}_{y x} & \bar{c}_{y y}+\bar{s}_{y}
\end{array}\right]
$$

The presence of cross terms in the modal damping matrix indicates that the FA and SS vibrations are coupled through damping. This coupling originates from the fact that the inclusion of the aerodynamic damping terms in $\mathbf{C}_{\text {Aero }}$ makes the system damping matrix $\overline{\boldsymbol{C}}$ non-proportional and therefore it is not diagonalized by the undamped modal shapes. Unlike traditional damping matrices, $\bar{c}_{x y}$ and $\bar{c}_{y x}$ are not identical, making the modal damping matrix asymmetric. This is not uncommon when considering damping in rotating machineries [26]. The 2-DOF model described by Equation (7) is an approximate model of the original one described by Equation (3) with only the first bending modes considered. Higher modes could be included in the model by using the relevant mode shapes and generalised coordinates in a similar way. The response of the turbine can be calculated by summing up the contributions from the modes considered. When the inflow wind field is uniform in space and constant in time, the aerodynamic damping matrix described by Equation (8) is constant during the simulation. However, when a turbulent inflow wind field is considered, the aerodynamic damping matrix becomes time-varying.

\section{Model verification and identification}

\subsection{Comparison between 2-DOF model and FAST}

For given operating conditions, the $2 \times 2$ modal aerodynamic damping matrix $\overline{\mathbf{C}}$ was calculated using Equation (8) with the airfoil properties provided with the FAST model. The stiffness and mass matrices for the 2-DOF model were computed from the material and geometric properties provided with the FAST model to make the models comparable. The 2-DOF model was verified against the FAST model by comparing the FA and SS responses at the tower top. To quantify the degree of correlation between the two responses, the Time Response Assurance Criterion (TRAC) [27] was used. Considering two time response column vectors $\mathbf{u}_{1}(t)$ and $\mathbf{u}_{2}(t)$ generated using different models, the TRAC is defined as

$$
T R A C=\frac{\left[\mathbf{u}_{1}(t)^{\mathrm{T}} \mathbf{u}_{2}(t)\right]^{2}}{\left[\mathbf{u}_{1}(t)^{T} \mathbf{u}_{1}(t)\right]\left[\mathbf{u}_{2}(t)^{T} \mathbf{u}_{2}(t)\right]},
$$

where $\mathbf{u}_{1}(t)$ and $\mathbf{u}_{2}(t)$ have the same duration and time step. Choosing the mean wind speed as $20 \mathrm{~m} / \mathrm{s}$, the rotor rotation speed is set to $12.1 \mathrm{rpm}$ and blade pitch angle 
to 17 degrees, giving the rated power output. The wind field was divided into a $11 \times 11$ rectangular grid with $20 \mathrm{~m}$ distance between two adjacent grid points. The centre of the grid is at $90 \mathrm{~m}$ height, close to the tower top at $87.6 \mathrm{~m}$. To better control the input wind field in both the 2-DOF model and the FAST model, the non-uniform and time-varying wind field was generated by a customised turbulent wind field generator in MATLAB, producing similar wind time series compared to the FAST wind field generator TurbSim [28]. Kaimal spectrum and relevant parameters (e.g. coherence length parameters) were selected as recommended by IEC 61400 - Edition 3 [29]. The turbulence intensity was selected as $10 \%$. In the FAST model, the following default settings were changed to allow a consistent comparison with our model. The non-uniform inflow wind field was used as the input; the tower was allowed to vibrate in the FA and SS directions, but the wind-tower interaction was not included (no shadow from the blades); the blades were set as rigid. For the 2-DOF model, the external modal forces were derived from the aerodynamic forces applied to the rigid tower considering the same inflow field as the FAST model. The coefficients in the time-varying aerodynamic damping matrix were calculated at every time step during the time integration.

The HHT- $\alpha$ method [30] was implemented with and without an extra Newton-Raphson step when the damping matrix is updated at every time step and the results were practically indistinguishable. From comparison results not shown here, the responses from the 2-DOF model with the time-varying damping matrix were very close to those from the FAST model with TRAC values of 1.000 for both the FA and SS response in this case. These values represent a baseline for good agreement against which others can be compared. Then the time-varying aerodynamic damping matrix was changed to a constant aerodynamic damping matrix by assuming the wind speed in the rotor plane equals the mean wind speed. Figure 2 compares the responses from the 2-DOF model with the constant aerodynamic damping matrix and those from the FAST model for a wind speed of $20 \mathrm{~m} / \mathrm{s}$, indicating that the FA and SS responses from these two models are close, with TRAC values of 1.000 for the FA response and 0.995 for the SS response. Figure 2(d) shows that the SS responses have similar frequencies but slightly different amplitudes. Similar comparison was conducted for wind speeds from $6 \mathrm{~m} / \mathrm{s}$ to $20 \mathrm{~m} / \mathrm{s}$, showing good agreement between the responses from the 2-DOF model and the FAST model (minimum TRAC values are 1.000 and 0.990 for the FA and SS responses respectively). These comparisons demonstrate that the constant aerodynamic damping matrix can adequately represent the damping characteristics of the wind turbine for non-uniform and 
time-varying wind fields. This shows that if the constant damping matrix can be identified, the dynamic behaviour of the system is captured accurately.

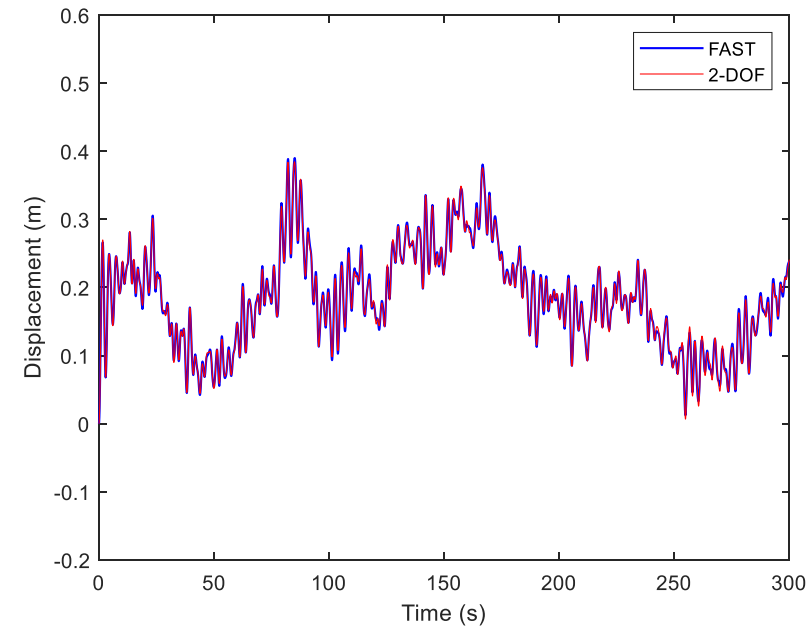

(a)

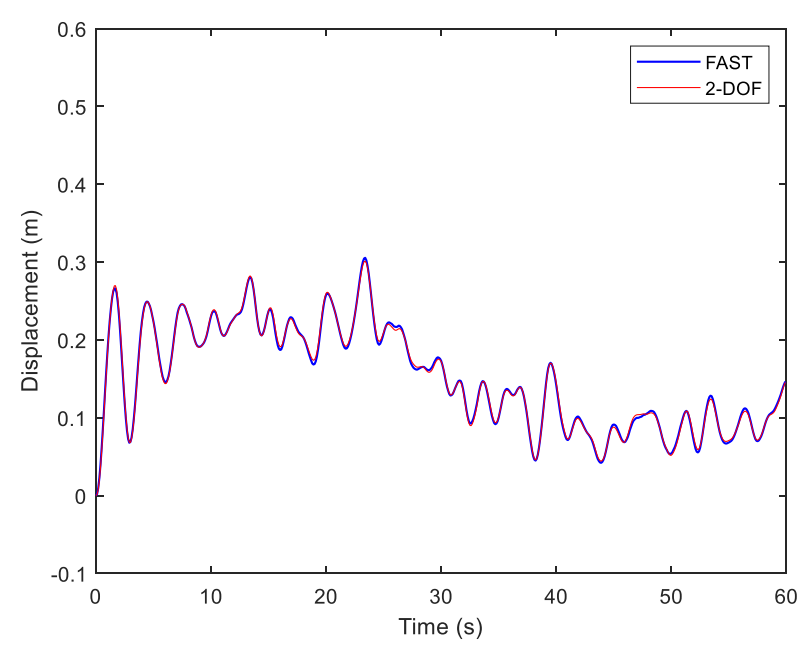

(c)

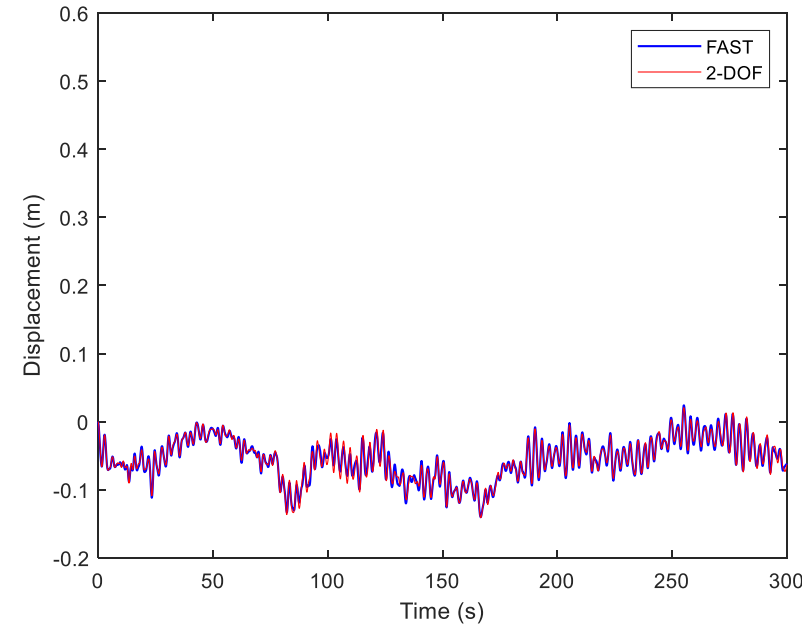

(b)

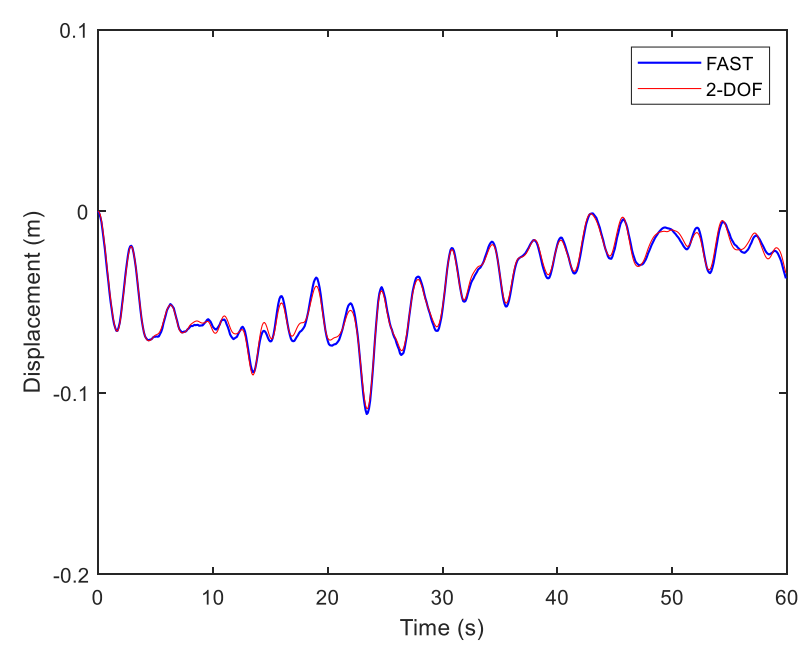

(d)

Figure 2. Comparison of the FA (a, c) and SS (b, d) responses from the 2-DOF model with the constant aerodynamic damping matrix and the FAST model; (c) and (d) are zooms of (a) and (b) over the initial 60s.

\subsection{Comparison between time series obtained using different damping models}

Many researchers have given analytical expressions for the FA damping [31], with Valamanesh and Myers [32] and [16] providing analytical expressions for both the FA and SS damping. Comparison was made between the responses generated by the 2-DOF model with the constant aerodynamic damping matrix described in section 2 and models using diagonal damping matrices made of aerodynamic damping ratios estimated 
following the methods provided in [32] and [16] (both produce identical damping ratios). For a mean wind speed of $20 \mathrm{~m} / \mathrm{s}$, the response comparison is shown in Figure 3. The FA and SS responses generated from the model with damping ratios are both different from those from the 2-DOF model, with a TRAC value of 0.984 in the FA direction and a TRAC value of 0.826 in the SS direction. From Section 3.1 we know that the 2-DOF model generates similar responses in both FA and SS directions compared to those from FAST. Both the FA and SS damping are under-estimated using the methods from [32] and [16], as the coupling between the FA and SS motions is not considered in the damping calculation formulae.

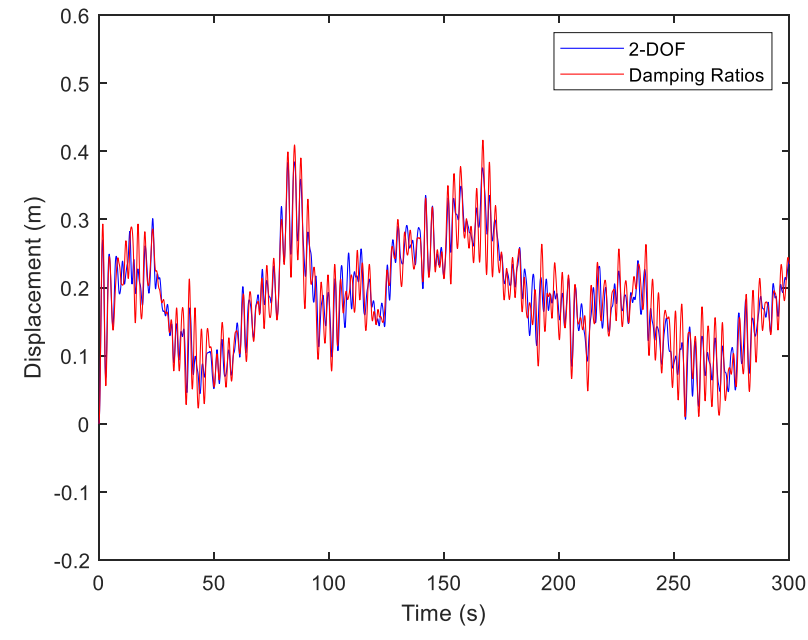

(a)

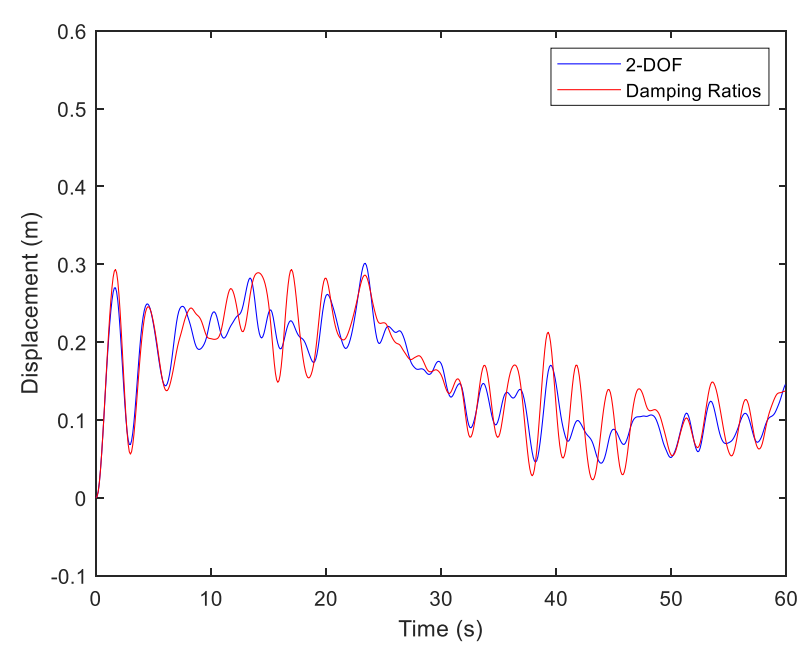

(c)

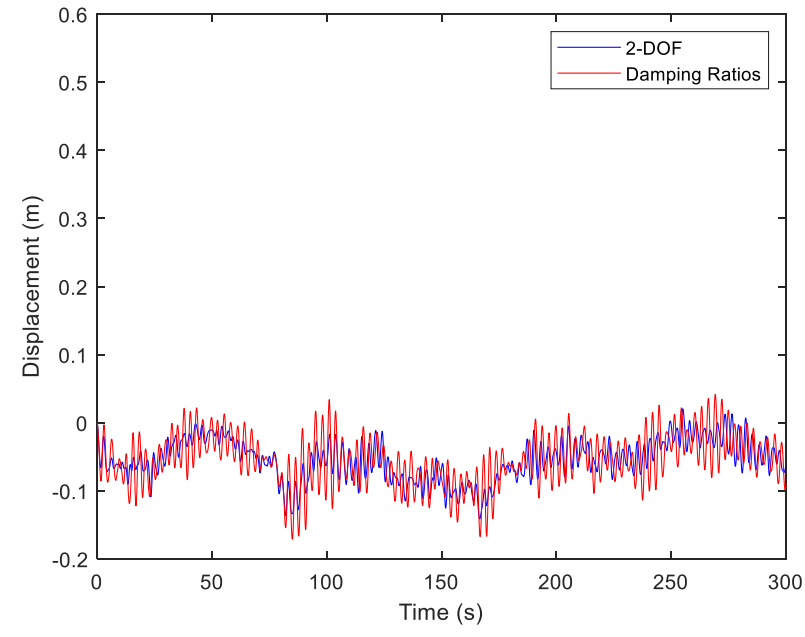

(b)

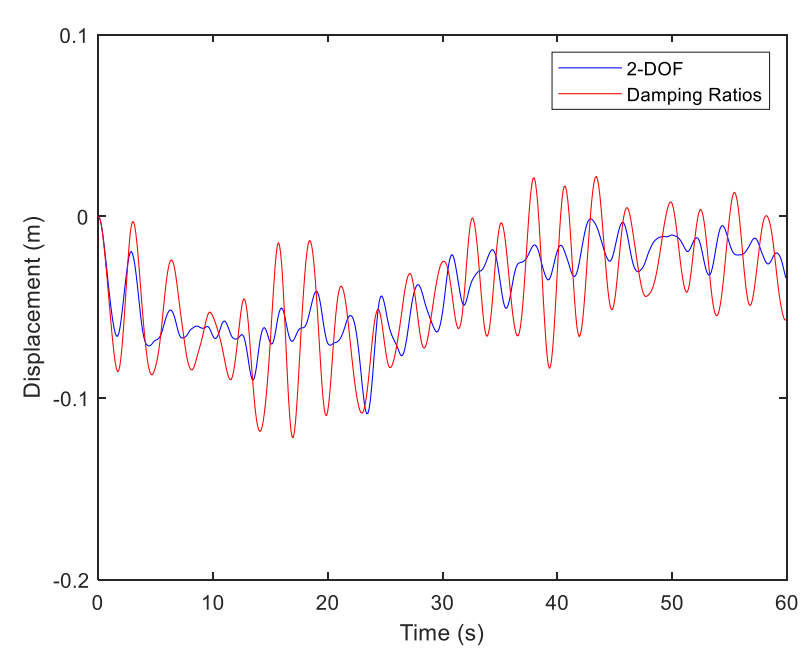

(d)

Figure 3. Comparison of the FA (a, c) and SS (b, d) responses from the 2-DOF model with the constant aerodynamic damping matrix and the model with estimated damping ratios; (c) and (d) are zooms of (a) and (b) over the initial 60s. 


\subsection{Damping identification using classic and modified OMA}

Two existing OMA methods were chosen to identify the damping ratios in the FA and SS directions. The first OMA method is classic data-driven SSI, and the second method is a modified SSI method [18]. The modified SSI method introduces a harmonic vector into the measurements in order to isolate structural modes from the response. It was adopted herein to reduce the influence induced by the interaction in the two directions, i.e., FA and SS, through taking the first mode vibration in one direction as a harmonic excitation. Based on the fundamental frequency $(0.34 \mathrm{~Hz})$ of the wind turbine (Table 1), the modified measurement was constructed as

$$
\mathbf{u}_{m}(t)=\mathbf{u}(t)+\mathbf{I} \sin (0.34 \cdot 2 \pi t),
$$

where $\mathbf{u}(t)$ is the wind turbine response, and $\mathbf{I}$ is an identity vector (all diagonal elements equal to 1 and otherwise zero) with the same size as $\mathbf{u}(t)$.

With the mean wind speed increasing from $6 \mathrm{~m} / \mathrm{s}$ to $20 \mathrm{~m} / \mathrm{s}$ ( $2 \mathrm{~m} / \mathrm{s}$ increment), turbulent non-uniform wind fields were generated by the customised turbulent wind field generator. For every mean wind speed, 10 random seeds were allocated to generate 10 sets of wind fields. These wind fields were used as input to the FAST model and corresponding wind turbine responses were obtained. The two OMA methods were used to separately estimate the damping ratios in the FA and SS directions. The mean values and the standard deviations of the damping ratio for all mean wind speeds can be obtained using the 10 data sets. The mean values of the damping ratios can be converted into damping coefficients using the modal stiffness and mass with regard to the first bending modes. These mean values of the damping coefficients and their standard deviations are plotted in Figure 4, together with the analytical diagonal damping coefficients in the 2-DOF model. The identified FA and SS damping coefficients using modified SSI are very close to those using classic SSI. The identified FA damping coefficients using both OMA methods are slightly lower than the analytical diagonal FA damping coefficients, showing a similar trend with wind speed. However, the identified SS damping coefficients using either OMA methods are much higher than the actual damping coefficient in the SS direction and do not show the increase with wind speed. 


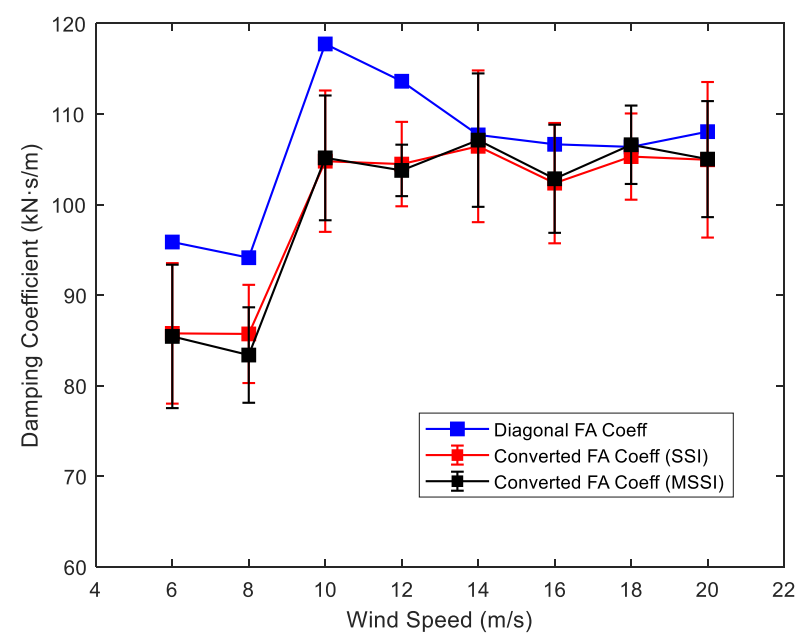

(a)

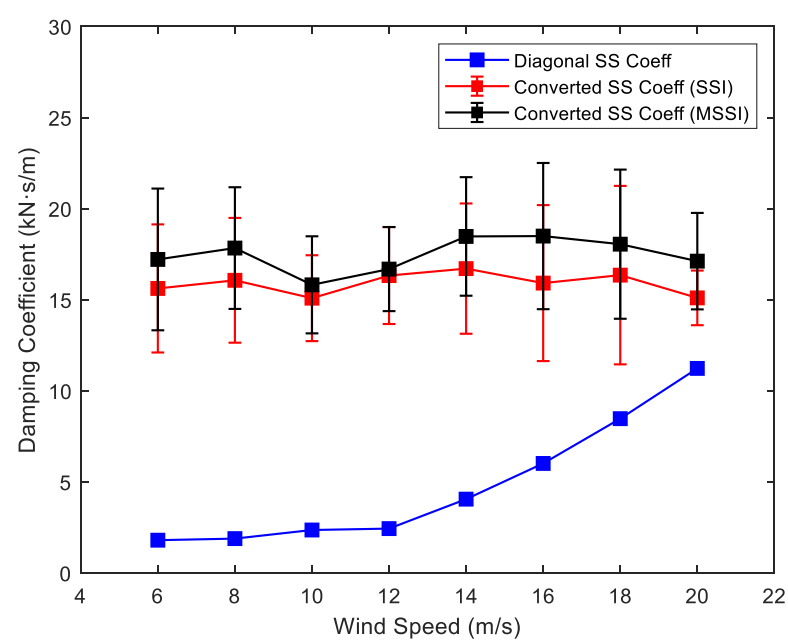

(b)

Figure 4. Comparison of the FA (a) and SS (b) identified damping coefficients using classic and modified SSI compared to theorectical diagonal damping matrix coefficients for wind speeds between 6 and $20 \mathrm{~m} / \mathrm{s}$.

For a wind speed of $20 \mathrm{~m} / \mathrm{s}$, the FA and SS responses generated from the model with the identified damping ratios using modified SSI are compared with the responses from FAST in Figure 5 with the same input forces. The TRAC values for the FA and SS responses are 1.000 and 0.941 . In the FA direction, the model with the identified damping coefficients produces similar responses compared to FAST. However, the responses in the SS direction generated by the 2-DOF model with identified damping ratios are quite different from those obtained from FAST. Setting the off-diagonal terms in the damping matrix in Equation (7) to zero, the dynamic system is effectively decoupled in the FA and SS directions. The agreement in the FA responses indicates that the offdiagonal terms do not significantly influence the FA motion and damping. The difference in the SS direction means that the identified damping ratios are not sufficient to describe the SS damping and that the off-diagonal terms have significant influence on the SS motion. 


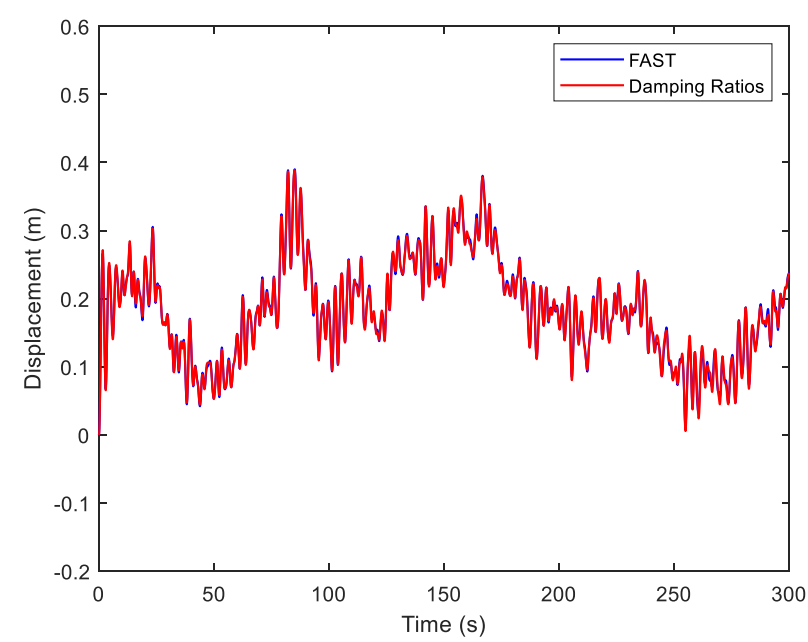

(a)

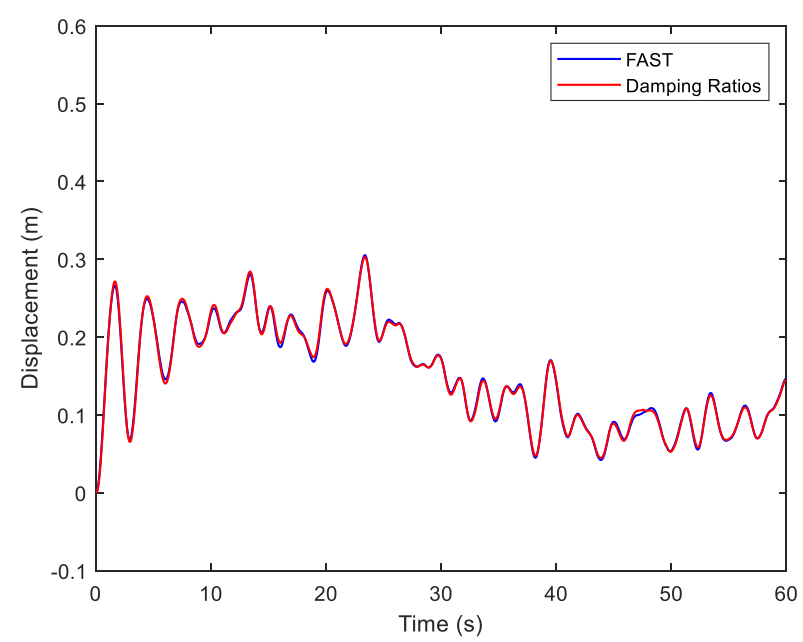

(c)

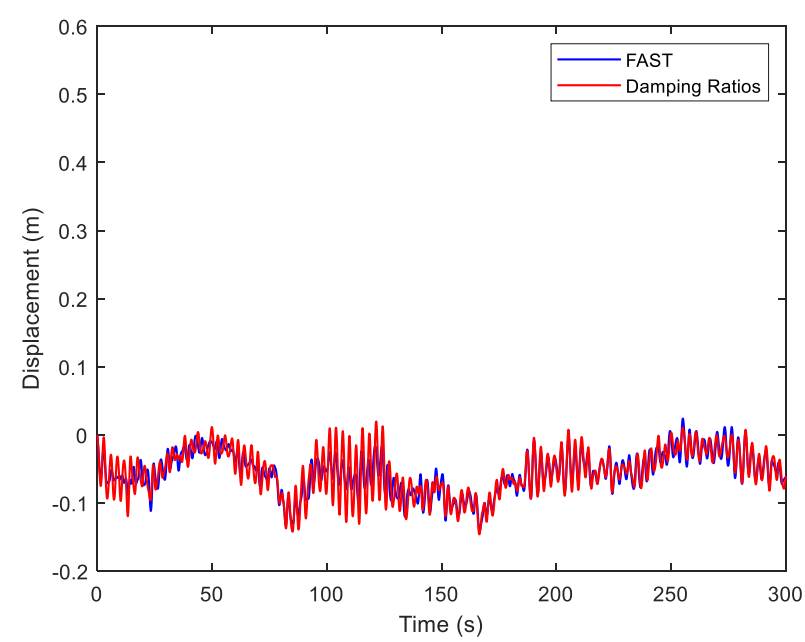

(b)

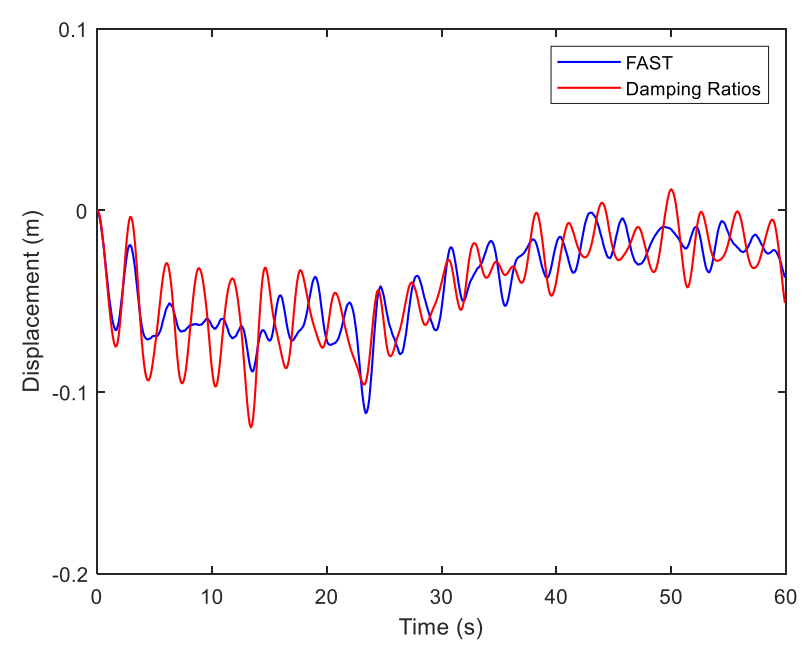

(d)

Figure 5. Comparison of the FA (a, c) and SS (b, d) responses from the FAST model and the model with identified damping ratios using modified SSI for $20 \mathrm{~m} / \mathrm{s}$ wind speed; (c) and (d) are zooms of (a) and (b) over the initial 60s.

Overall, the results from this section show that the 2-DOF model generates similar responses for the same input as the FAST model, confirming that the modal damping matrix in the 2-DOF model describes the aerodynamic damping in the wind turbine system adequately. On the other hand, the models with traditional aerodynamic damping ratios obtained analytically or using OMA cannot describe the SS vibration of the turbine system well enough, requiring identification of the aerodynamic damping matrix. 


\section{Damping identification}

\subsection{Methodology}

In this section the objective is to identify the unconventional damping in the system from simulated time series data for the FA and SS directions. One possibility for doing this would be to identify the complex modes directly ([33][34]). However, here we opted to directly identify the reduced aerodynamic damping matrix $\overline{\mathbf{C}}$ defined in Equation (8) as the compounded effect of closely spaced first bending modes and the nonproportional aerodynamic damping would be mode complexity [33]. Identifying the $2 \times 2$ damping matrix targets the aerodynamic component directly. The general damping identification matrix method proposed by Chen et al. [35] was used. The method, outlined in Appendix B, estimates the damping matrix from the Frequency Response Functions (FRFs) of the structure and assumes that the mass and stiffness matrices are already known (Appendix C). Although originally presented for standard system matrices, the assumptions underpinning this method are applicable to the problem of identifying the damping matrix defined here. Converting the wind turbine model to a 2-DOF system as described in Section 2 significantly reduces the FRFs to be estimated to a $2 \times 2$ matrix $\mathbf{H}(\omega)$ for the first bending modes. The FRF matrix for the 2-DOF system can be written as

$$
\mathbf{H}(\omega)=\left[\begin{array}{ll}
H_{x x}(\omega) & H_{x y}(\omega) \\
H_{y x}(\omega) & H_{y y}(\omega)
\end{array}\right],
$$

where for example the term $H_{x y}$ represents the transfer function between the output FA displacement $x$ and an input force in the $y$ (SS) direction (other terms follow this format). Once $\mathbf{H}(\omega)$ is obtained, $\mathbf{G}(\omega)$ can be calculated $(\mathbf{G}(\omega)=$ $-\operatorname{Im}(\mathbf{H}(\omega))[\operatorname{Re}(\mathbf{H}(\omega))]^{-1}$ see Appendix B). The modal stiffnesses and masses, and the mode shapes in Equation (7) can be obtained by traditional OMA methods on a parked turbine, from which $\mathbf{H}^{N}(\omega)=\left[\mathbf{K}-\omega^{2} \mathbf{M}\right]^{-1}$ can be determined. Then, following Chen et al. [35], the damping matrix can be determined by averaging the calculated frequency-dependent damping terms.

From this summary, the success of Chen's identification method hinges on a satisfactory estimation of the relevant transfer functions. The FRF matrix in this study is obtained using responses caused by the combination of wind loading and harmonic forces applied at the tower top. Initially, it was attempted to calculate the input force based on the incoming mean wind speed and BEM calculations, but this gave poor results both 
in terms of FRF and damping estimations, necessitating the use a harmonic force at the tower top. This may present practical challenges, but is closer to conventional experimental modal analysis [5]. As the response of an operating wind turbine is caused by the combination of the aerodynamic loading and the harmonic excitation at a particular frequency, the harmonic excitation must be large enough so that it produces a discernible component at that frequency in the response spectrum. The synthetic times series used for the FRF "measurement" were output from the open-source package OpenFAST by NREL [36]. Additional code was written into the source code of OpenFAST to allow harmonic forces to be applied at the tower top with different frequencies and amplitudes. These forces can be applied separately in the FA/SS directions. The modified OpenFAST code was then compiled using Microsoft Visual Studio 2015 to generate a new FAST executable file, from which the dynamic responses of wind turbines can be obtained and used to calculate the FRF matrix.

\subsection{Identification results with harmonic forces}

For the mean wind speed equal to $20 \mathrm{~m} / \mathrm{s}$, using the processes described in Section 2, the modal properties and aerodynamic modal damping matrix of the 2-DOF model were obtained. The calculated aerodynamic damping matrix is

$$
\left[\begin{array}{cc}
108.1 & 21.3 \\
41.4 & 11.2
\end{array}\right](k N \cdot s / m)
$$

which is the target to identify.

The harmonic force amplitude was set to $10 \mathrm{kN}$ (sensitivity to this setting is discussed later) and the harmonic excitation frequency varied from $0.2 \mathrm{~Hz}$ to $0.5 \mathrm{~Hz}$, as this range contains the resonance frequency and allowed to obtain stable averaged damping coefficients. The frequency increment for the harmonic forces was $0.01 \mathrm{~Hz}$, except for the range from $0.3 \mathrm{~Hz}$ to $0.38 \mathrm{~Hz}$ where a smaller frequency increment of $0.004 \mathrm{~Hz}$ was chosen to obtain higher resolutions around the resonance frequency $(0.34 \mathrm{~Hz})$. The forces were separately applied to the FA and SS directions together with the aerodynamic forces caused by the non-uniform wind field and corresponding responses recorded. The FRF was obtained from the FFT of steady state responses divided by the FFT of the applied harmonic forces. The real parts of identified FRF curves are compared with those calculated analytically in Figure 6. A small frequency shift was found around the resonance frequency, but for frequencies away from the resonance good agreement between the analytical FRF curves and the identified curves was obtained. 
Calculation of the four aerodynamic damping coefficients was carried out from the frequency-dependent estimations of these coefficients. The averaged damping matrix estimation is

$$
\left[\begin{array}{cc}
108.5 & 20.3 \\
44.4 & 11.5
\end{array}\right](k N \cdot s / m)
$$

which is close to the analytical values with small percentage differences:

$$
\left[\begin{array}{cc}
0.4 & -4.6 \\
7.3 & 2.2
\end{array}\right](\%)
$$

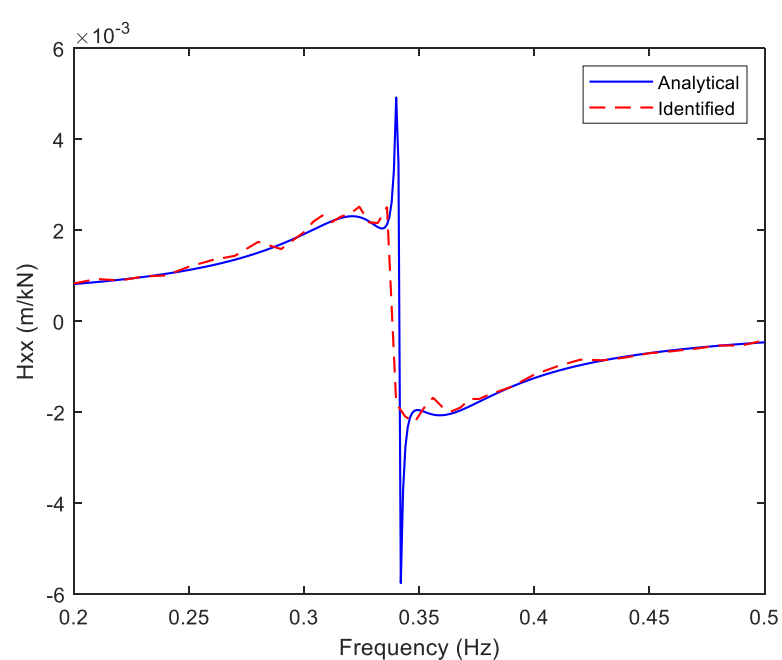

(a)

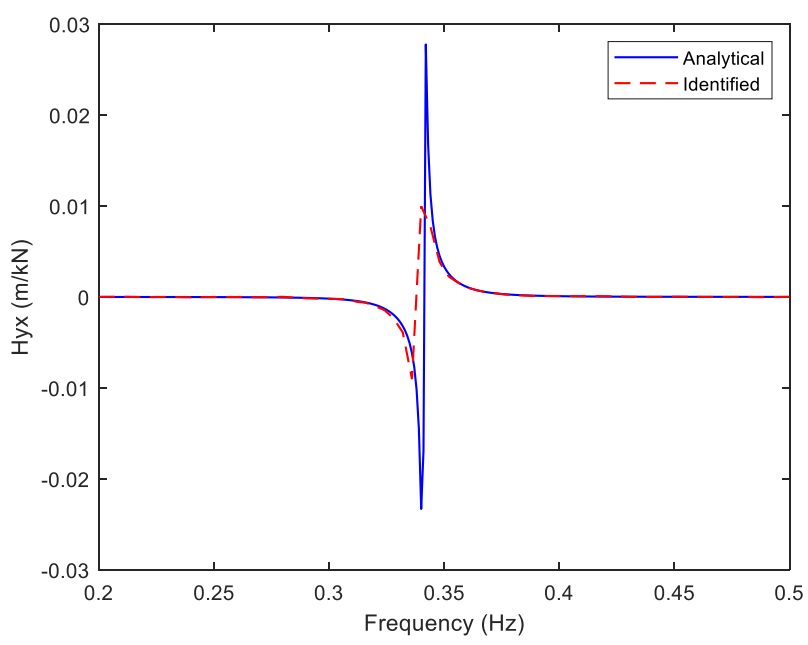

(c)

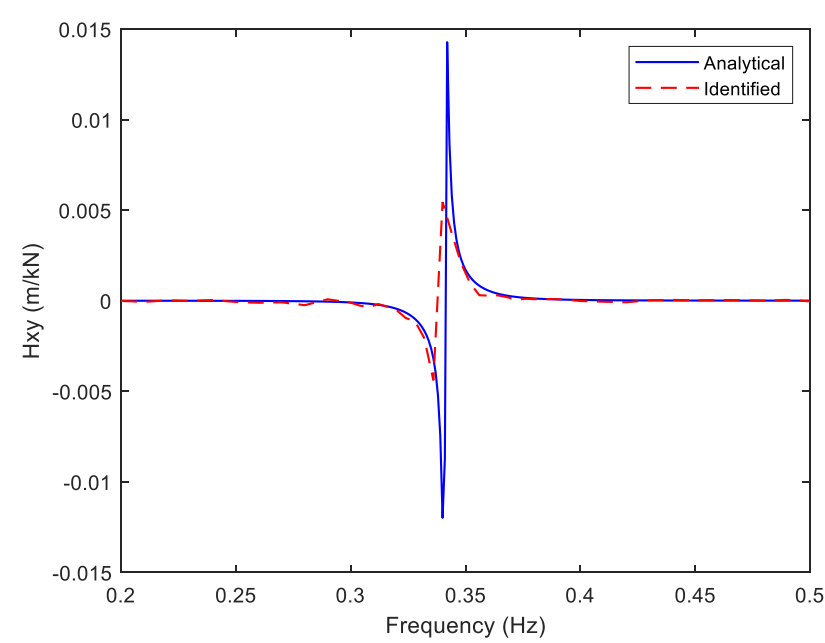

(b)

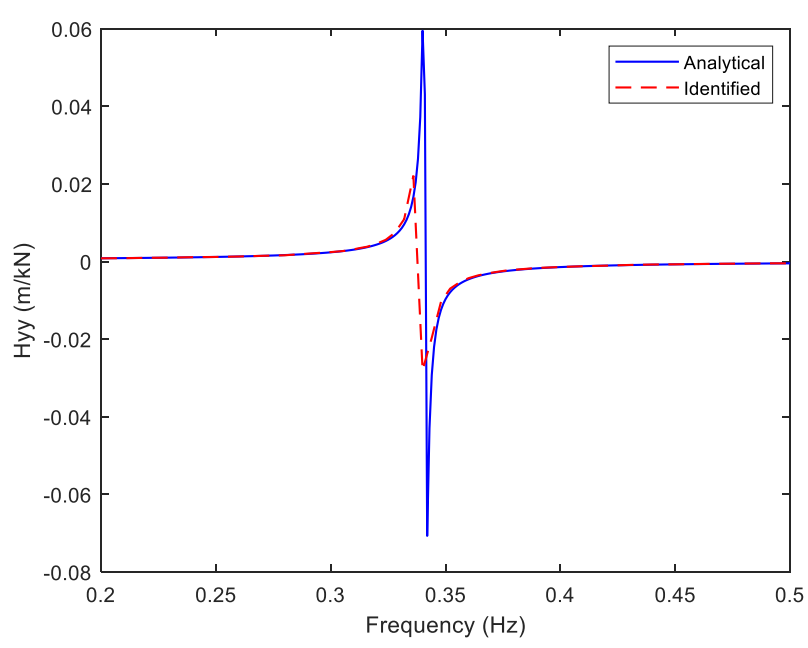

(d)

Figure 6. Comparison the real part of analytical and identified $H_{x x}$ (a), $H_{x y}$ (b), $H_{y x}$ (c) and $H_{y y}(\mathrm{~d})$ with with harmonic excitations. 


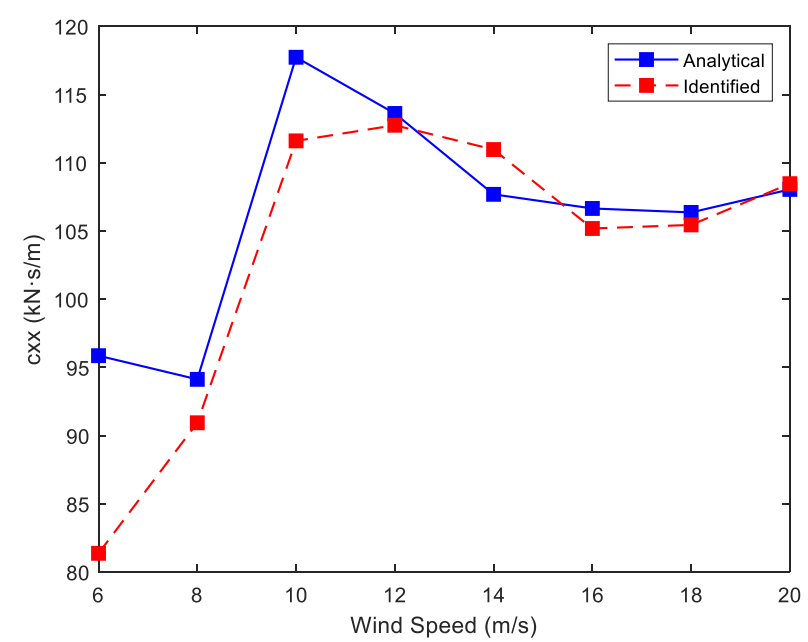

(a)

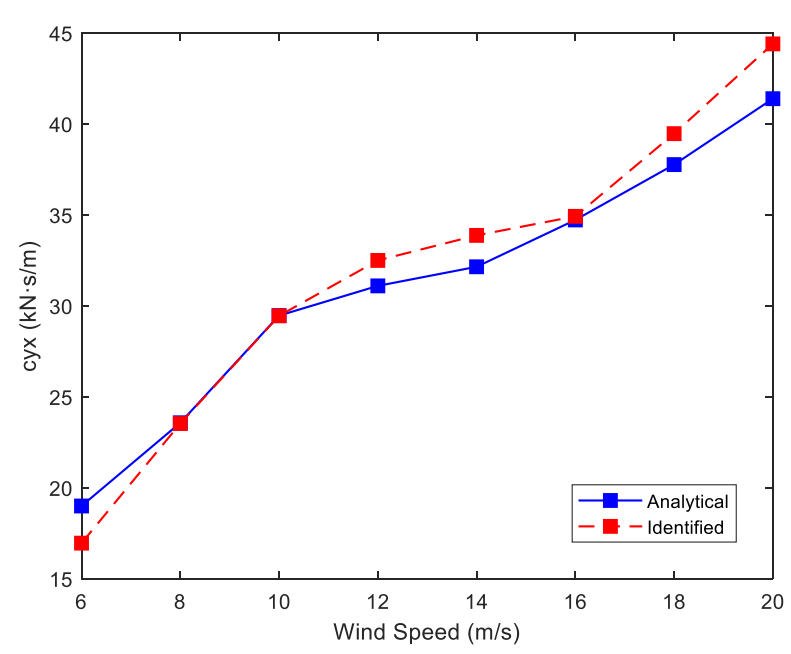

(c)

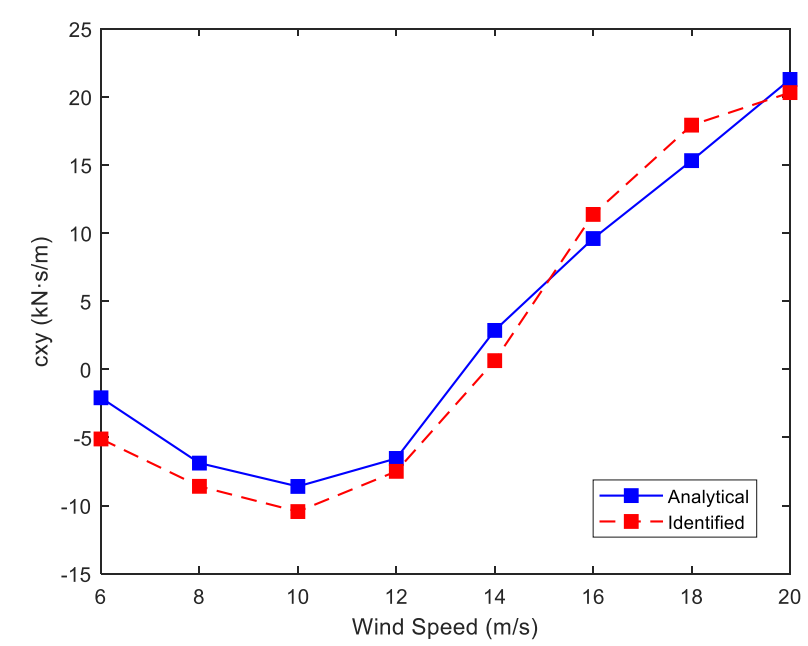

(b)

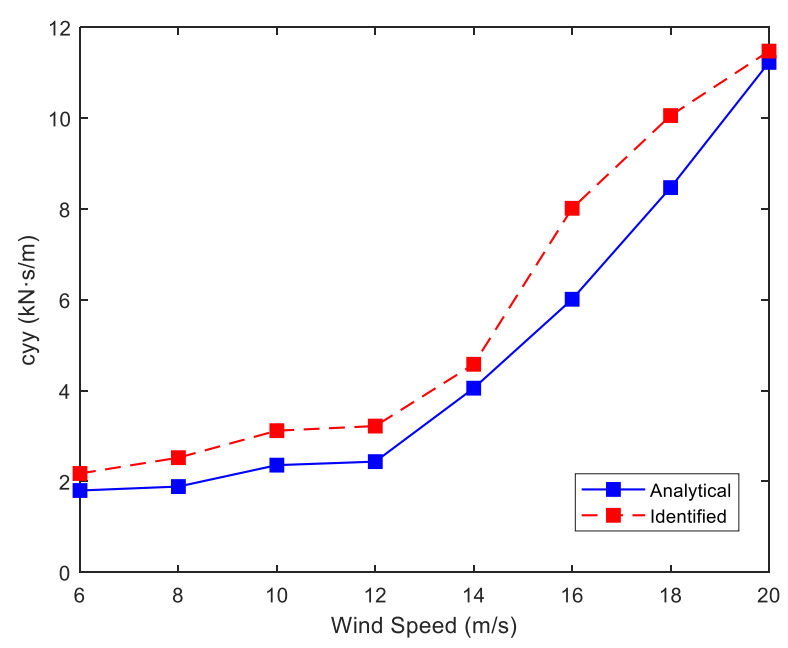

(d)

Figure 7. Comparison of the analytical and identified damping matrix coefficients $\bar{c}_{x x}$ (a), $\bar{c}_{x y}$ (b); $\bar{c}_{y x}$ (c) and $\bar{c}_{y y}$ (d) with with harmonic force excitation $(10 \mathrm{kN})$ for mean wind speeds from $6 \mathrm{~m} / \mathrm{s}$ to $20 \mathrm{~m} / \mathrm{s}$ in $2 \mathrm{~m} / \mathrm{s}$ steps.

The identification with harmonic excitations was undertaken for non-uniform wind fields with mean wind speeds from $6 \mathrm{~m} / \mathrm{s}$ to $20 \mathrm{~m} / \mathrm{s}$ in $2 \mathrm{~m} / \mathrm{s}$ steps. The identified results compared with analytical values are shown in Figure 7, confirming the generally good performance of the identification procedure using harmonic excitations. The percentage differences for the estimations of $\bar{c}_{x x}$ and $\bar{c}_{y x}$ are less than $10 \%$, while for $\bar{c}_{y y}$ the percentage differences are less than $30 \%$. For $\bar{c}_{x y}$, most percentage differences are around $20 \%$. The accuracy of this method depends on the amplitude of the harmonic excitation, with larger harmonic forces giving more accurate results since in the 
frequency domain the corresponding response due to the harmonic excitation is distinct and easier to identify. When the amplitude of the harmonic excitation is too low, it is hard to obtain FRFs accurate enough to achieve good estimation of the damping matrix. For the $5 \mathrm{MW}$ reference onshore turbine used in this study, when the amplitudes of the harmonic excitation are lower than $5 \mathrm{kN}$, the damping matrix estimation is poor. Tests were also carried out with harmonic excitations with amplitudes of $100 \mathrm{kN}$, from which the estimation of the FRFs and the damping matrix is more accurate. For example, when the mean wind speed is $20 \mathrm{~m} / \mathrm{s}$, the identified damping coefficients $\bar{c}_{x x}, \bar{c}_{x y}$ and $\bar{c}_{x y}$ have a percentage difference around $2 \%$ compared to the analytical values, while for the estimation of $\bar{c}_{y y}$ the percentage difference is $7 \%$. However, in practice it would be difficult to apply large excitations of amplitude $100 \mathrm{kN}$. The selection of the force amplitude depends on the size, inertia and stiffness of the wind turbine, with smaller wind turbines requiring smaller forces for the identification based on harmonic excitations. In practice, besides shakers to excite wind turbines, active tuned mass dampers could be used to harmonically excite the wind turbine (if installed), and this method was recommended by Oh and Ishihara [37]. 


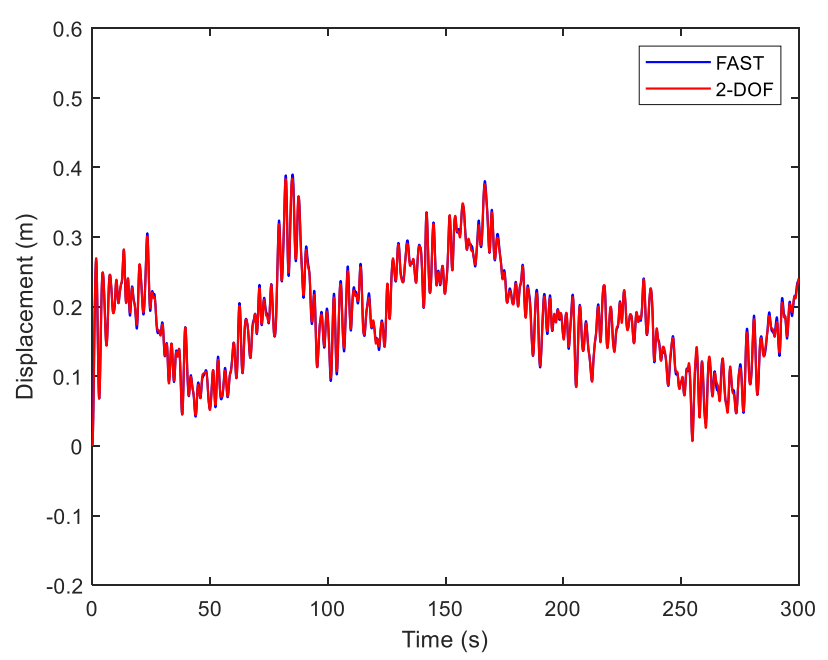

(a)

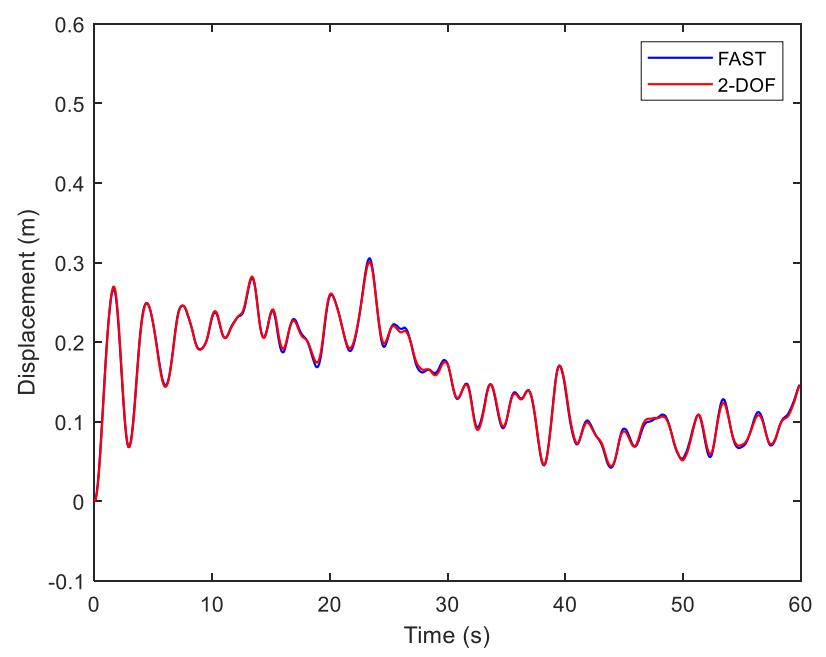

(c)

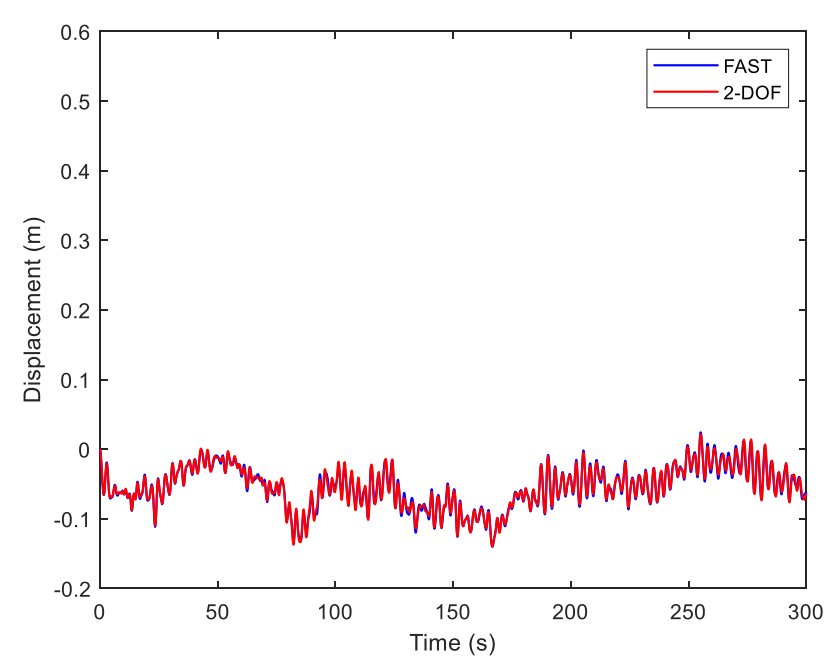

(b)

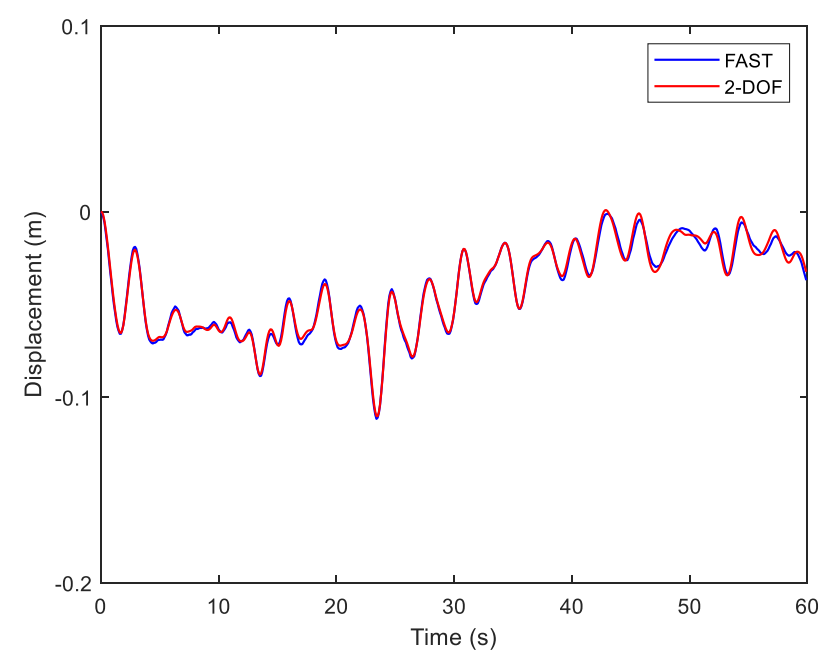

(d)

Figure 8. Comparison of the FA (a, c) and SS (b, d) responses from the FAST model and the model with identified damping ratios using 2 DOF matrix with harmonic excitation; (c) and (d) are zooms of (a) and (b).

Using the identified damping matrix for $20 \mathrm{~m} / \mathrm{s}$ mean wind speed to define the damping in the 2-DOF model, the generated responses are compared with those from FAST in Figure 8. The comparison shows that for both FA and SS directions the 2-DOF model with the identified damping matrix is able to generate similar responses compared to FAST, with TRAC values of 1.000 and 0.996 for the FA and SS directions respectively. Together with results from Section 3, these results demonstrate that the identified aerodynamic damping matrix allows the SS dynamic behaviour to be captured much more 
accurately than when damping ratios identified using either the classic or modified SSI are used. As different identification methods and measured responses were used to obtain the damping ratios in Figure 5 and the modal damping matrix in Figure 8, it is not straightforward to compare the performance. However, it should be noted that the identified damping ratios do not represent all of the damping mechanisms in the system, i.e., the cross-coupling between FA and SS motions.

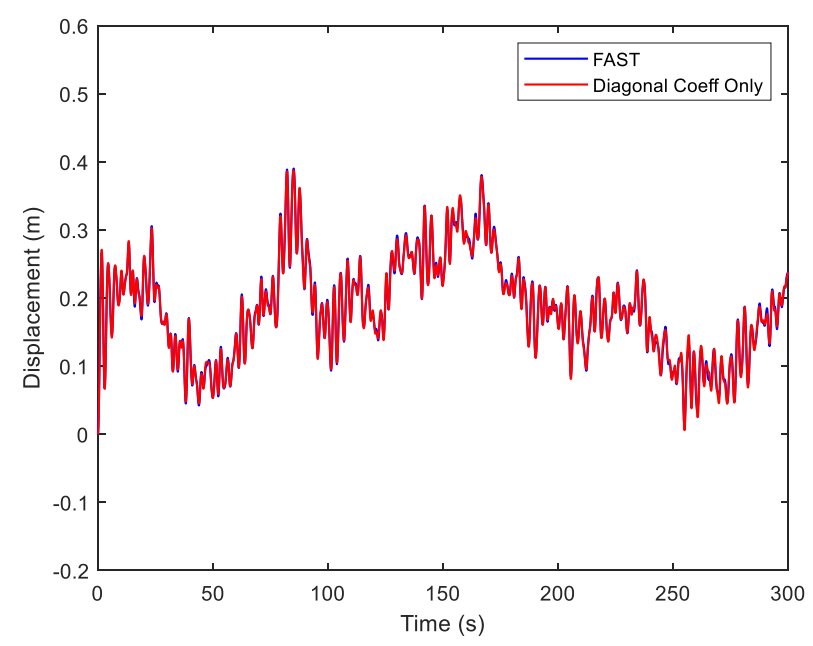

(a)

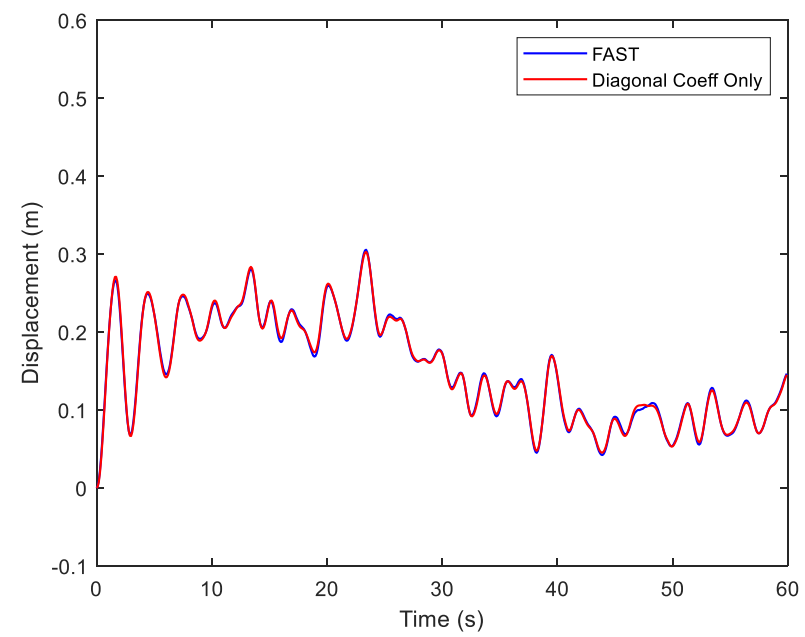

(c)

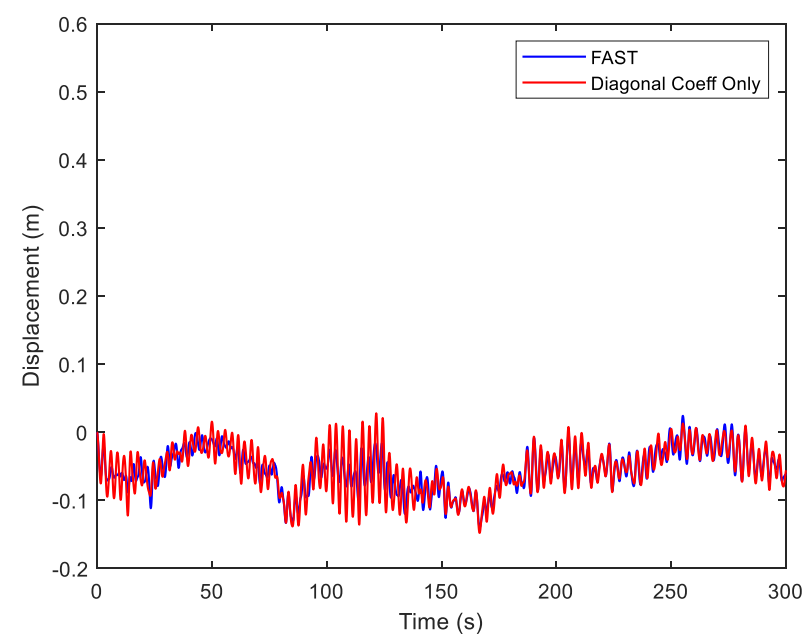

(b)

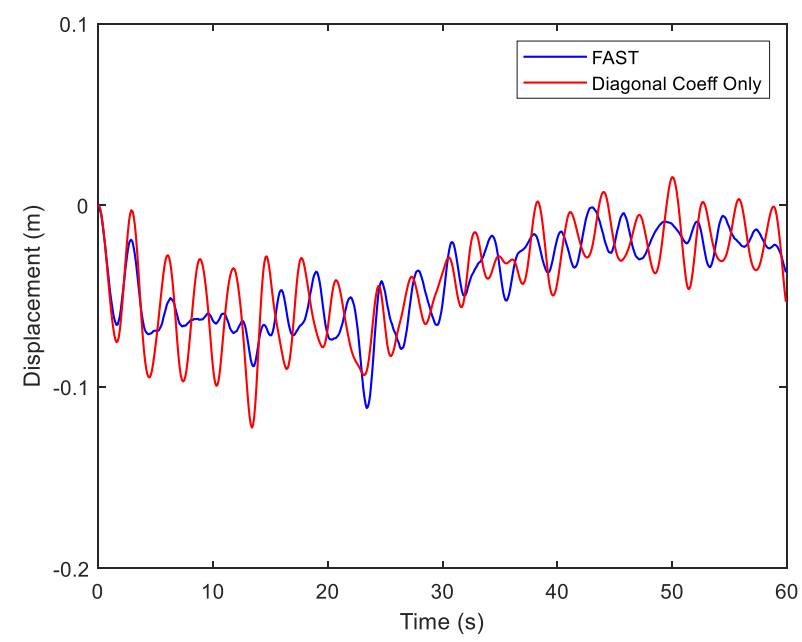

(d)

Figure 9. Comparison of the FA $(a, c)$ and SS $(b, d)$ responses from the FAST model and the model with only diagonal damping coefficients; (c) and (d) are zooms of (a) and (b). 
As a final check, the off-diagonal terms in the aerodynamic damping matrix in the 2DOF model were set to zero, keeping only the diagonal terms in the damping in the matrix. This simulates a perfect identification of the diagonal damping ratios but removes the coupling between the FA and SS. With the same wind field $(20 \mathrm{~m} / \mathrm{s}$ mean speed), the responses in the FA and SS directions from the 2-DOF model with only diagonal terms were computed and compared with those from FAST. The results are shown in Figure 9, and give TRAC values 1.000 and 0.928 for the FA and SS responses, respectively. This comparison shows that the FA responses are still very close, while the SS responses are quite different. From these results we can conclude that the full damping matrix, including the coupling between FA and SS direction is necessary to describe the vibration behaviour in the SS direction. Ignoring this coupling will lead to inaccurate predictions however accurate the identification of the diagonal damping terms is. Identifying damping ratios using OMA is standard practice and as such it was the intention of this section to see how the simulated dynamic behaviour of the system using these damping ratios compares to that obtained from the proposed damping model.

\section{Conclusions}

This paper proposes a novel methodology to identify the aerodynamic damping of wind turbines in operation. The study is based on a FE model representing the tower and simplified wind-rotor interaction based on blade element momentum calculations. The proposed identification technique is based on:

1. Aerodynamic damping model developed in an earlier publication that captures the damping through a non-symmetric damping matrix.

2. An unconventional reduction of the complete numerical model to a 2-DOF system that is shown to capture well the dynamic behaviour of the system and was verified against FAST simulation results. Using this reduction limits the task of identification to a fully populated $2 \times 2$ damping matrix.

3. Extending an existing FRF matrix-based damping identification (Chen et al. [35]).

The transfer functions were obtained by applying harmonic force inputs in the FA and SS directions at the top of the tower (similar to traditional EMA under shaker 
excitation). Identification results obtained from the proposed method were compared to existing standard and modified SSI based OMA techniques. The conclusions are:

- The FA damping ratios can be identified well by all methods;

- Existing OMA methods only identify the SS damping ratios with significant error;

- The proposed new identification method can extract the $2 \times 2$ damping matrix with good accuracy, provided a force input with sufficient amplitude is applied.

- In practice, the size of devices to generate external, harmonic forces needs careful consideration for large wind turbine structures.

- Identifying the full $2 \times 2$ damping matrix including the coupling between FA and SS is essential to correctly capture the vibration behaviour in the SS direction.

\section{Acknowledgement}

The author(s) disclosed receipt of the following financial support for the research, authorship and/or publication of this article: The first author gratefully acknowledges the financial support from the State's Key Project of Research and Development Plan (No.2016YFE0127900). The first author also gratefully acknowledges the helpful discussions with Prof. Xugang Hua from Hunan University.

\section{References}

[1] Rezaei R, Fromme P, Duffour P. Fatigue life sensitivity of monopile-supported offshore wind turbines to damping. Renew Energy 2018; 123: 450-459.

[2] Martinez-Luengo M, Kolios A, Wang L. Structural health monitoring of offshore wind turbines: A review through the Statistical Pattern Recognition Paradigm. Renew Sustain Energy Rev 2016; 64: 91-105.

[3] Shirzadeh R, Devriendt C, Bidakhvidi MA, et al. Experimental and computational damping estimation of an offshore wind turbine on a monopile foundation. J Wind Eng Ind Aerodyn 2013; 120: 96-106.

[4] Hansen MH, Thomsen K, Fuglsang P, et al. Two methods for estimating aeroelastic damping of operational wind turbine modes from experiments. Wind Energy 2006; 9: 179-191. 
[5] Ewins DJ. Modal testing: theory, practice and application. Second Edi. Research Studies Press LTD., 2000.

[6] Koukoura C, Natarajan A, Vesth A. Identification of support structure damping of a full scale offshore wind turbine in normal operation. Renew Energy 2015; 81: 882-895.

[7] Osgood R, Bir G, Mutha H, et al. Full-scale modal wind turbine tests: comparing shaker excitation with wind excitation. In: Proceedings of the 28th International Modal Analysis Conference. Jacksonville, Florida, 2010.

[8] Ozbek M, Meng F, Rixen DJ. Challenges in testing and monitoring the inoperation vibration characteristics of wind turbines. Mech Syst Signal Process 2013; 41: 649-666.

[9] Devriendt C, Magalhaes F, Weijtjens W, et al. Structural health monitoring of offshore wind turbines using automated operational modal analysis. Struct Heal Monit 2014; 13: 644-659.

[10] Bajrić A, Høgsberg J, Rüdinger F. Evaluation of damping estimates by automated Operational Modal Analysis for offshore wind turbine tower vibrations. Renew Energy 2018; 116: 153-163.

[11] Ozbek M, J. Rixen D. Operational modal analysis of a 2.5 MW wind turbine using optical measurement techniques and strain gauges. Wind Energy 2013; 17: 367-381.

[12] Hansen MH. Aeroelastic stability analysis of wind turbines using an eigenvalue approach. Wind Energy 2004; 7: 133-143.

[13] Weijtjens W, Rasoul Shirzadeh, Gert De Sitter, et al. Classifying resonant frequencies and damping values of an offshore wind turbine on a monopile foundation for different operational conditions. In: Proceedings of the European Wind Energy Association. Barcelona, Spain, 2014.

[14] $\mathrm{Hu} \mathrm{W}$, Thöns S, Rohrmann RG, et al. Vibration-based structural health monitoring of a wind turbine system. Part I: Resonance phenomenon. Eng Struct 2015; 89: 260-272.

[15] Tcherniak D, Chauhan S, Hansen MH. Applicability limits of operational modal analysis to operational wind turbines. In: Proceedings of the 28th International Modal Analysis Conference. Jacksonville, Florida, 2010.

[16] Chen C, Duffour P. Modelling damping sources in monopile-supported offshore wind turbines. Wind Energy 2018; 21: 1121-1140. 
[17] James III GH, Carne TG, Lauffer, et al. The natural excitation technique (NExT) for modal parameter extraction from operating wind turbines. Int J Anal Exp Modal Anal 1993; 10: 260-277.

[18] Dai K, Wang Y, Huang Y, et al. Development of a modified stochastic subspace identification method for rapid structural assessment of in-service utility-scale wind turbine towers. Wind Energy 2017; 17: 657-669.

[19] Dong X, Lian J, Yang M, et al. Operational modal identification of offshore wind turbine structure based on modified stochastic subspace identification method considering harmonic interference. J Renew Sustain Energy 2014; 6: 033128.

[20] Chen C, Duffour P, Fromme P. Modelling wind turbine tower-rotor interaction through an aerodynamic damping matrix. J Sound Vib 2020; 115667.

[21] Jonkman JM, Buhl MLJ. FAST user's guide. Technical Report NREL/EL-50038230. National Renewable Energy Laboratory: Golden, CO, USA, October 2005.

[22] Jonkman JM, Butterfield S, Musial W, et al. Definition of a 5-MW reference wind turbine for offshore system development. Technical Report NREL/TP-50038060. National Renewable Energy Laboratory: Golden, CO, USA, 2009.

[23] Hansen M. Aerodynamics of wind turbines. Second Edi. Routledge, 2008.

[24] Shirzadeh R, Weijtjens W, Guillaume P, et al. The dynamics of an offshore wind turbine in parked conditions: A comparison between simulations and measurements. Wind Energy 2014; 18: 1685-1702.

[25] Craig RR, Kurdila AJ. Fundamentals of structural dynamics. Second Edi. John Wiley \& Sons Ltd.

[26] Gutiérrez-Wing ES. Modal analysis of rotating machinery structures. PhD Thesis. Imperial College London, 2003.

[27] Avitabile P, Pingle P. Prediction of full field dynamic strain from limited sets of measured data. Shock Vib 2012; 19: 765-785.

[28] Jonkman BJ, Kilcher L. TurbSim user's guide: version 1. 06.00. Technical Report. National Renewable Energy Laboratory: Golden, CO, USA, 2012.

[29] International Electrotechnical Commission (IEC). IEC 61400-3 Wind turbines Part 3: Design requirements for offshore wind turbines. 2009.

[30] Gavin HP. Numerical integration in structural dynamics. Duke University, http://people.duke.edu/ hpgavin/cee541/NumericalIntegration.pdf (2016). 
[31] Salzmann DJC, Tempel J Van Der. Aerodynamic damping in the design of support structures for offshore wind turbines. In: The European Offshore Wind Conference \& Exhibition. Copenhagen, Denmark, 2005.

[32] Valamanesh V, Myers AT. Aerodynamic damping and seismic response of horizontal axis wind turbine towers. J Struct Eng 2014; 140: 1-9.

[33] Imregun M, Ewins DJ. Complex modes-origins and limits. Proc 13th Int Modal Anal Conf 1995; 496-506.

[34] Peeters B, Van der Auweraer H. PolyMAX: A revolution in operational modal analysis. Proc 1st Int Oper Modal Anal Conf IOMAC 2005.

[35] Chen SY, Ju MS, Tsuei, YG. Estimation of mass, stiffness and damping matrices from frequency response functions. $J$ Vib Acoust 1996; 118: 78-82.

[36] National Renewable Energy Laboratory (NREL). FAST v8 and the transition to OpenFAST, $\quad$ https://wind.nrel.gov/nwtc/docs/README_OpenFAST.pdf (2017).

[37] Oh S, Ishihara T. Structural parameter identification of a $2.4 \mathrm{MW}$ bottom fixed wind turbine by excitation test using active mass damper. Wind Energy 2018; 21: $1232-1238$. 


\section{Appendix A}

To consider a non-uniform inflow wind field, some modifications are needed to the derivation process in Section 3.3 of reference [20]. Keeping the initial assumptions that the rotor is rigid and the RNA speed is small, the aerodynamic forces applied to one blade element can still be expressed by Equations (4) to (7) in [20]. However, when summing the elemental blade forces for one blade, the three blades must be considered individually as the total aerodynamic forces experienced by different blades are different due to wind turbulence and different azimuthal positions. In this way, the aerodynamic force resultants can still be linearized as forces applied to a rigid tower plus terms related to the tower top velocities. Using the notations introduced in [20], the total force at the tower top in the $x$ (FA) direction can be expressed as the sum of thrusts applied to all blades:

$$
\begin{aligned}
& F_{x}^{\text {Flex }}(t)=\sum_{i=1}^{N_{b}} \int_{0}^{R} d T\left(V_{x R e l}, V_{r R e l}\right) \\
& =\sum_{i=1}^{N_{b}} \int_{0}^{R} d T\left(V_{0}, V_{r}\right)-\dot{x} \sum_{i=1}^{N_{b}} \int_{0}^{R} \frac{\partial(d T)}{\partial V_{0}}-\dot{\theta}_{y} \sum_{i=1}^{N_{b}} \cos \gamma_{i}(t) \int_{0}^{R} r \frac{\partial(d T)}{\partial V_{0}} \\
& -\dot{y} \sum_{i=1}^{N_{b}} \cos \gamma_{i}(t) \int_{0}^{R} \frac{\partial(d T)}{\partial V_{r}}+\dot{\theta}_{x} \sum_{i=1}^{N_{b}} \int_{0}^{R} r \frac{\partial(d T)}{\partial V_{r}}
\end{aligned}
$$

The total force in the $y$ (SS) direction is:

$$
\begin{aligned}
& F_{y}^{\text {Flex }}(t)=-\sum_{i=1}^{N_{b}} \cos \gamma_{i}(t) \int_{0}^{R} d S\left(V_{x R e l}, V_{r R e l}\right) \\
& =-\sum_{i=1}^{N_{b}} \cos \gamma_{i}(t) \int_{0}^{R} d S\left(V_{0}, V_{r}\right)+\dot{x} \sum_{i=1}^{N_{b}} \cos \gamma_{i}(t) \int_{0}^{R} \frac{\partial(d S)}{\partial V_{0}} \\
& +\dot{\theta}_{y} \sum_{i=1}^{N_{b}} \cos ^{2} \gamma_{i}(t) \int_{0}^{R} r \frac{\partial(d S)}{\partial V_{0}}+\dot{y} \sum_{i=1}^{N_{b}} \cos ^{2} \gamma_{i}(t) \int_{0}^{R} \frac{\partial(d S)}{\partial V_{r}} \\
& -\dot{\theta}_{x} \sum_{i=1}^{N_{b}} \cos _{i}(t) \int_{0}^{R} r \frac{\partial(d S)}{\partial V_{r}} .
\end{aligned}
$$

The total moment about the $x$ axis is:

$$
M_{x}^{\text {Flex }}(t)=\sum_{i=1}^{N_{b}} \int_{0}^{R} d M_{x}\left(V_{x R e l}, V_{r R e l}\right)
$$




$$
\begin{gathered}
\sum_{i=1}^{N_{b}} \int_{0}^{R} r d S\left(V_{0}, V_{r}\right)-\dot{x} \sum_{i=1}^{N_{b}} \int_{0}^{R} r \frac{\partial(d S)}{\partial V_{0}} \\
-\dot{\theta}_{y} \sum_{i=1}^{N_{b}} \cos \gamma_{i}(t) \int_{0}^{R} r^{2} \frac{\partial(d S)}{\partial V_{0}} \\
-\dot{y} \sum_{i=1}^{N_{b}} \cos _{i}(t) \int_{0}^{R} r \frac{\partial(d S)}{\partial V_{r}}+\dot{\theta}_{x} \sum_{i=1}^{N_{b}} \int_{0}^{R} r^{2} \frac{\partial(d S)}{\partial V_{r}}
\end{gathered}
$$

whereas the total moment about the $y$ axis is:

$$
\begin{aligned}
& M_{y}^{\text {Flex }}(t)=\sum_{i=1}^{N_{b}} \int_{0}^{R} d M_{y}\left(V_{x R e l}, V_{r R e l}\right) \\
& =\sum_{i=1}^{N_{b}} \cos \gamma_{i}(t) \int_{0}^{R} r d T\left(V_{0}, V_{r}\right)-\dot{x} \sum_{i=1}^{N_{b}} \cos \gamma_{i}(t) \int_{0}^{R} r \frac{\partial(d T)}{\partial V_{0}} \\
& -\dot{\theta}_{y} \sum_{i=1}^{N_{b}} \cos ^{2} \gamma_{i}(t) \int_{0}^{R} r^{2} \frac{\partial(d T)}{\partial V_{0}}-\dot{y} \sum_{i=1}^{N_{b}} \cos ^{2} \gamma_{i}(t) \int_{0}^{R} r \frac{\partial(d T)}{\partial V_{r}} \\
& +\dot{\theta}_{x} \sum_{i=1}^{N_{b}} \cos _{i}(t) \int_{0}^{R} r^{2} \frac{\partial(d T)}{\partial V_{r}} .
\end{aligned}
$$

The derivatives in Equations (A. 1) to (A. 4) can be found using expressions of partial derivatives in Appendix A in [20]. According to Equations (A. 1) to (A. 4), the resultant aerodynamic forces from the rotor to the top of a flexible wind turbine tower, $\mathbf{F}_{\text {Flex }}^{\text {Top }}(t)=\left[F_{x}^{\text {Flex }}(t) F_{y}^{\text {Flex }}(t) M_{x}^{\text {Flex }}(t) M_{y}^{\text {Flex }}(t)\right]^{T}$, can be rewritten in the following simplified form

$$
\mathbf{F}_{\text {Flex }}^{\text {Top }}(t)=\left[\begin{array}{c}
\sum_{i=1}^{N_{b}} \int_{0}^{R} d T\left(V_{0}, V_{r}\right) \\
-\sum_{i=1}^{N_{b}} \cos \gamma_{i}(t) \int_{0}^{R} d S\left(V_{0}, V_{r}\right) \\
\sum_{i=1}^{N_{b}} \int_{0}^{R} r d S\left(V_{0}, V_{r}\right) \\
\sum_{i=1}^{N_{b}} \cos \gamma_{i}(t) \int_{0}^{R} r d T\left(V_{0}, V_{r}\right)
\end{array}\right]-\mathbf{C}_{\text {Aero } 0}\left[\begin{array}{c}
\dot{x} \\
\dot{y} \\
\dot{\theta}_{x} \\
\dot{\theta}_{y}
\end{array}\right]=\mathbf{F}_{\text {Rigid }}^{\text {Top }}(t)-\mathbf{C}_{\text {Aero }} \dot{\mathbf{u}}^{\text {Top }}(t) .
$$


where

$$
\begin{gathered}
\mathbf{C}_{\text {Aero }}= \\
{\left[\begin{array}{cccc}
\sum_{i=1}^{N_{b}} \int_{0}^{R} \frac{\partial(d T)}{\partial V_{0}} & \sum_{i=1}^{N_{b}} \cos \gamma_{i}(t) \int_{0}^{R} \frac{\partial(d T)}{\partial V_{r}} & -\sum_{i=1}^{N_{b}} \int_{0}^{R} r \frac{\partial(d T)}{\partial V_{r}} & \sum_{i=1}^{N_{b}} \cos \gamma_{i}(t) \int_{0}^{R} r \frac{\partial(d T)}{\partial V_{0}} \\
-\sum_{i=1}^{N_{b}} \cos \gamma_{i}(t) \int_{0}^{R} \frac{\partial(d S)}{\partial V_{0}} & -\sum_{i=1}^{N_{b}} \cos ^{2} \gamma_{i}(t) \int_{0}^{R} \frac{\partial(d S)}{\partial V_{r}} & \sum_{i=1}^{N_{b}} \cos \gamma_{i}(t) \int_{0}^{R} r \frac{\partial(d S)}{\partial V_{r}} & -\sum_{i=1}^{N_{b}} \cos ^{2} \gamma_{i}(t) \int_{0}^{R} r \frac{\partial(d S)}{\partial V_{0}} \\
\sum_{i=1}^{N_{b}} \int_{0}^{R} r \frac{\partial(d S)}{\partial V_{0}} & \sum_{i=1}^{N_{b}} \cos \gamma_{i}(t) \int_{0}^{R} r \frac{\partial(d S)}{\partial V_{r}} & -\sum_{i=1}^{N_{b}} \int_{0}^{R} r^{2} \frac{\partial(d S)}{\partial V_{r}} & \sum_{i=1}^{N_{b}} \cos \gamma_{i}(t) \int_{0}^{R} r^{2} \frac{\partial(d S)}{\partial V_{0}} \\
\sum_{i=1}^{N_{b}} \cos \gamma_{i}(t) \int_{0}^{R} r \frac{\partial(d T)}{\partial V_{0}} & \sum_{i=1}^{N_{b}} \cos ^{2} \gamma_{i}(t) \int_{0}^{R} r \frac{\partial(d T)}{\partial V_{r}} & -\sum_{i=1}^{N_{b}} \cos \gamma_{i}(t) \int_{0}^{R} r^{2} \frac{\partial(d T)}{\partial V_{r}} & \sum_{i=1}^{N_{b}} \cos ^{2} \gamma_{i}(t) \int_{0}^{R} r^{2} \frac{\partial(d T)}{\partial V_{0}}
\end{array}\right] .}
\end{gathered}
$$

$\mathbf{C}_{\text {Aero }}$ can be written more concisely:

$$
\mathbf{C}_{\text {Aero }}=\left[\begin{array}{cccc}
c_{x x} & c_{x y} & c_{x \theta_{x}} & c_{x \theta_{y}} \\
c_{y x} & c_{y y} & c_{y \theta_{x}} & c_{y \theta_{y}} \\
c_{\theta_{x} x} & c_{\theta_{x} y} & c_{\theta_{x} \theta_{x}} & c_{\theta_{x} \theta_{y}} \\
c_{\theta_{y} x} & c_{\theta_{y} y} & c_{\theta_{y} \theta_{x}} & c_{\theta_{y} \theta_{y}}
\end{array}\right] .
$$




\section{Appendix}

\section{Chen et al.'s method [35]}

Given a dynamic system with mass, stiffness and damping matrices $\mathbf{M}, \mathbf{K}$ and $\mathbf{C}$ excited by an external force $\mathbf{f}$, the equation of motion is

$$
\mathbf{M} \ddot{\mathbf{x}}+\mathbf{C} \dot{\mathbf{x}}+\mathbf{K x}=\mathbf{f}
$$

Rewrite this equation of motion in the frequency domain:

$$
\left(-\omega^{2} \mathbf{M}+i \omega \mathbf{C}+\mathbf{K}\right) \mathbf{X}(\omega)=\mathbf{F}(\omega)
$$

The frequency response function (FRF) matrix $\mathbf{H}(\omega)$ is defined as:

$$
\mathbf{H}(\omega)=\left(-\omega^{2} \mathbf{M}+i \omega \mathbf{C}+\mathbf{K}\right)^{-1}
$$

The "normal" FRF $\mathbf{H}^{\mathbf{N}}(\omega)$ is defined with the undamped system:

$$
\mathbf{H}^{\mathrm{N}}(\omega)=\left[\mathbf{K}-\omega^{2} \mathbf{M}\right]^{-1}
$$

With the "normal" FRF, the frequency domain equation of motion can be written as

$$
\left[\mathbf{H}^{\mathrm{N}}(\omega)\right]^{-1} \mathbf{X}(\omega)+i \omega \mathbf{C X}(\omega)=\mathbf{F}(\omega)
$$

or

$$
\mathbf{X}(\omega)+i \mathbf{G}(\omega) \mathbf{X}(\omega)=\mathbf{H}^{N}(\omega) \mathbf{F}(\omega)
$$

where

$$
\mathbf{G}(\omega)=\omega \mathbf{H}^{N}(\omega) \mathbf{C}
$$

Therefore, the relationship between the measured FRF $\mathbf{H}(\omega)$ and the "normal" FRF $\mathbf{H}^{\mathrm{N}}(\omega)$ is

$$
\mathbf{H}^{N}(\omega)=[\mathbf{I}+i \mathbf{G}(\omega)] \mathbf{H}(\omega)
$$

where $\mathbf{I}$ is an identity matrix. Since $\mathbf{H}^{N}(\omega)$ and $\mathbf{G}(\omega)$ are real matrices, the imaginary part of the RHS in the above equation is zero, giving

$$
\mathbf{G}(\omega)=-\operatorname{im}(\mathbf{H}(\omega))[\operatorname{Re}(\mathbf{H}(\omega))]^{-1}
$$

Finally, the damping matrix at any given frequency can be expressed by

$$
\mathbf{C}=\frac{1}{\omega}\left[\mathbf{H}^{\mathbf{N}}(\omega)\right]^{-1} \mathbf{G}(\omega)
$$

It should be noted that this damping identification method requires prior knowledge of the stiffness and mass matrices. 


\section{Appendix C}

Using the modal decomposition in Section 2.2, the corresponding 2-DOF model can be obtained. The modal mass matrix is

$$
\left[\begin{array}{cc}
1834 & 0 \\
0 & 1834
\end{array}\right](\mathrm{kN} / \mathrm{m}) .
$$

The modal stiffness matrix is

$$
\left[\begin{array}{cc}
3990 & 0 \\
0 & 3900
\end{array}\right]\left(k N \cdot s^{2} / m\right) .
$$

The modal stiffness and mass matrices for the 2-DOF model were assumed known before the estimation of damping matrix. 